\title{
Spherical accretion of a perfect fluid onto a black hole
}

\author{
F. S. Guzmán \\ Instituto de Física y Matemáticas, Universidad Michoacana de San Nicolás de Hidalgo, \\ Edificio C-3, Cd. Universitaria, 58040 Morelia, Michoacán, México. \\ A. Romero-Amezcua \\ Facultad de Ciencias Físico Matemáticas, Universidad Michoacana de San Nicolás de Hidalgo. \\ Edificio Alpha, Cd. Universitaria, 58040 Morelia, Michoacán, México. \\ I. Alvarez-Ríos \\ Instituto de Física y Matemáticas, Universidad Michoacana de San Nicolás de Hidalgo. \\ Edificio C-3, Cd. Universitaria, 58040 Morelia, Michoacán, México.
}

Received 16 July 2020; accepted 24 December 2020

\begin{abstract}
In this academic paper we present in detail the numerical solution of the accretion of a perfect flui onto a black hole. The conditions are very simple, we consider a radial flu being accreted by a Schwarzschild black hole. We present two scenarios: 1) the test fiel case in which the flui does not affect the geometry of the black hole space-time background, and 2) the full non-linear scenario, in which the geometry of the space-time evolves simultaneously with the flui according to Einstein's equations. In the two scenarios we describe the black hole space-time in horizon penetrating coordinates, so that it is possible to visualize that accretion actually takes place within the numerical domain. In the non-linear scenario we solve the equations of geometry using the ADM formulation of General Relativity, with very simple gauge and boundary conditions, and include diagnostics related to the apparent and event horizon growth. In view of the recent spectacular discoveries by the Event Horizon Telescope (EHT) collaboration and future discoveries, the aim of this paper is to provide a detailed description for interested students in Black Hole Astrophysics, of accretion onto black holes in the simplest scenario, which can be introductory to understand the much more realistic magnetohydrodynamics accretion solutions used to model the EHT observations.
\end{abstract}

Keywords: Numerical relativity; black holes; accretion; relativistic astrophysics.

PACS: 04.25.D-; 98.62.Mw; 04.25.dg; 04.70.-s

DOI: https://doi.org/10.31349/RevMexFisE.18.020206

\section{Introduction}

One of the outstanding recent breakthroughs in science is the capture of the firs image of a black hole by the Event Horizon Telescope collaboration. This result is a consequence of intense observational and theoretical efforts that brought together the largest array of telescopes ever, and the most sophisticated theoretical models and simulations of plasma dynamics around black holes [1].

The discovery and science around the image of black holes contain important contributions on matter modeling and data analysis for the transformation of observational data into accurate physical scenarios. Matter models involve magnetohydrodynamics (MHD) and radiation, coupled with different degrees of complexity. For example, the plasma around black holes in the EHT discovery, was supposed to be a gas obeying the MHD equations under a garden variety of magnetic fiel and environment conditions. With such a number of simulations, during a post-process analysis, a catalog of images was produced to compare with observations using image classificatio methods [1].

Simulations provide the solution of a direct problem for various combinations of gas and magnetic fiel configura tions, black hole masses and various other parameters, which are essential to construct an image catalog. The identificatio of the simulations of the catalog with the astronomical obser- vations define an inverse problem that determines the most likely values of the parameters used in simulations. For a description of a simple direct-inverse problem in the context of black hole images with simple flui conditions see e.g. [2]. In this academic paper we focus on the basics of flui dynamics of matter around black holes.

We illustrate the process of accretion considering the simplest scenario, namely a Schwarzschild black hole and a spherically symmetric accretion of a perfect fluid As simple as it is, all the involved technicalities form a considerable set of details worth being aware of. The idea in this paper is to be as detailed as possible, even if we deal with a simple scenario.

The problem is solved in two cases: 1) the test flui case, in which the matter does not affect the geometry of the spacetime, and 2) the full non-linear accretion that involves the evolution of the space-time, which needs the solution of Einstein's equations simultaneously with the fluid s equations, and illustrate the growth of the apparent and event horizon of the black hole during the accretion. We expect the nonlinear case to be the state of the art in the symbiosis between higher resolution observations and simulations in the near future within the EHT research, of course with the General Relativistic MHD which is considerably more complex than the case in this paper. 
The paper is organized as follows. In Sec. 2 we describe the evolution equations of a perfect flui on top of the black hole space-time background, so as the numerical methods used to solve them. In Sec. 3 we present the solution for the accretion process. In Sec. 4 we present the strategy to solve Einstein's equations for a spherically symmetric space-time using simple gauge and boundary conditions; we analyze a particular case in detail using important diagnostics of matter and space-time dynamics. Finally in Section $\mathrm{V}$ we mention a few fina comments.

\section{Hydrodynamics equations on a spherically symmetric space-time}

The construction of the flui dynamics and space-time geometry equations will be based on the $3+1$ decomposition of the space-time. The line element for a general space-time metric in this approach reads [3]

$$
d s^{2}=\left(-\alpha^{2}+\beta^{i} \beta_{i}\right) d t^{2}+2 \beta_{i} d t d x^{i}+\gamma_{i j} d x^{i} d x^{j},
$$

where $\alpha$ is the lapse, $\beta^{i}$ the shift vector, $\gamma_{i j}$ is the 3-metric of the spatial hypersurfaces of the space-time $\Sigma_{t}$, with $i, j$ labeling spatial coordinates and $\beta_{i}=\gamma_{i j} \beta^{j}$. The normal vector to $\Sigma_{t}$ at each point of the space-time is given by $n^{\mu}=g^{\mu \nu} n_{\nu}$, where $n_{\nu}=(-\alpha, 0,0,0)$ is the 1 -form measuring the density of hypersurfaces along the normal direction.

We consider the matter to be a perfect fluid whose stressenergy tensor for a general space-time with metric $g_{\mu \nu}$ is

$$
T^{\mu \nu}=\rho_{0} h u^{\mu} u^{\nu}+p g^{\mu \nu},
$$

where each volume element has rest mass density $\rho_{0}$, specifi enthalpy $h=1+e+p / \rho_{0}$, internal energy $e$, pressure $p$ and 4-velocity $u^{\mu}$.

With this metric one can construct expressions of the 4velocity of a flui element $u^{\mu}=\left(u^{0}, u^{i}\right)$ in terms of the velocities according to Eulerian observers:

$$
\begin{aligned}
u^{0} & =\frac{d t}{d \tau}=\frac{W}{\alpha}=\frac{1}{\alpha \sqrt{1-v^{i} v_{i}}}, \\
v^{i} & =\frac{u^{i}}{u^{0}}=\frac{u^{i}}{W}+\frac{\beta^{i}}{\alpha},
\end{aligned}
$$

where $t$ is the coordinate time associated to a flui element from an Eulerian reference frame, $\tau$ the proper time measured by the element, $v^{i}$ the components of its 3-velocity and $W$ the Lorentz factor where $v^{i} v_{i}=\gamma_{i j} v^{i} v^{j}$.

Relativistic hydrodynamics equations consist of the mass conservation and the divergence-free nature of the stressenergy tensor, whose covariant expressions are

$$
\begin{aligned}
\nabla_{\mu}\left(\rho_{0} u^{\mu}\right) & =0, \\
\nabla_{\mu} T^{\mu \nu} & =0,
\end{aligned}
$$

where $\nabla_{\mu}$ is the covariant derivative consistent with the metric $g_{\mu \nu}$, of the space-time, $x^{\mu}=x^{0}, x^{1}, x^{2}, x^{3}$ or $x^{\mu}=x^{0}, x^{i}$ are the coordinates of the space-time, where greek indices label space-time coordinates $0,1,2,3$, whereas latin indices 1, 2, 3 label space coordinates of the hypersurfaces $\Sigma_{t}$.

These equations are the Relativistic Euler equations, and can be cast in a flu balance set of laws based on the Valencia formulation $[4,5]$. These equations can be constructed if the conservation laws are written such that the divergence is applied on a vector fiel $\xi^{\mu}$, so that we can use the formula $\nabla_{\mu} \xi^{\mu}=(1 / \sqrt{-g}) \partial_{\mu}\left(\sqrt{-g} \xi^{\mu}\right)$. Equation (4) has already this form and can be expanded immediately. However equation (5) can be brought to this form by calculating the divergence of the projections of $T_{\mu \nu}$. For the projection one define the local basis of the space-time $\left\{\mathbf{e}_{0}, \mathbf{e}_{i}\right\}$, with $\mathbf{e}_{0}=n_{\mu}$ and $\mathbf{e}_{i}=\left(\beta_{i}, \gamma_{i j}\right)$, a basis such that $\mathbf{e}_{0} \cdot \mathbf{e}_{i}=0$. Then the divergence of the product $T^{\mu \nu} \mathbf{e}_{i \nu}$ is the divergence of the projection on the hypersurface $\Sigma_{t}$ and becomes three equations, whereas the divergence of $T^{\mu \nu} \mathbf{e}_{0 \nu}$ is the projection along the normal to $\Sigma_{t}$, whose divergence is the equation for the conservation of energy. With this in mind, equations (4) and (5) can be reduced to the following set of flu balance equations

$$
\frac{\partial \mathbf{u}}{\partial t}+\frac{\partial \mathbf{F}^{\mathbf{i}}(\mathbf{u})}{\partial x^{i}}=\mathbf{S}(\mathbf{u})
$$

with the following state vector $\mathbf{u}$, vector of flu es $\mathbf{F}$ and vector of sources $\mathbf{S}$ :

$$
\begin{aligned}
\mathbf{u} & =\left(\begin{array}{c}
D \\
J_{j} \\
\tau
\end{array}\right), \\
\mathbf{F}(\mathbf{u})= & \left(\begin{array}{c}
\alpha\left(v^{i}-\frac{\beta^{i}}{\alpha}\right) D \\
\alpha\left(v^{i}-\frac{\beta^{i}}{\alpha}\right) J_{j}+\alpha \sqrt{\gamma} p \delta_{j}^{i} \\
\alpha\left(v^{i}-\frac{\beta^{i}}{\alpha}\right) \tau+\alpha \sqrt{\gamma} p v^{i}
\end{array}\right), \\
\mathbf{S}(\mathbf{u})= & \left.\begin{array}{c}
0 \\
\alpha \sqrt{\gamma} T^{\mu \nu} g_{\nu \sigma} \Gamma_{\mu j}^{\sigma}{ }_{\mu j} \\
\alpha \sqrt{\gamma}\left(T^{\mu 0} \partial_{\mu} \alpha-\alpha T^{\mu \nu} \Gamma_{\mu \nu}^{0}\right)
\end{array}\right),
\end{aligned}
$$

for $i, j=1,2,3$, where the state vector entries are define in terms of the six primitive variables $\rho_{0}, v^{i}, e, p$ as

$$
\begin{aligned}
D & =\sqrt{\gamma} \rho_{0} W, \\
J_{i} & =\sqrt{\gamma} \rho_{0} h W^{2} v_{i}, \\
\tau & =\sqrt{\gamma}\left(\rho_{0} h W^{2}-p-\rho_{0} W\right),
\end{aligned}
$$

called the conservative variables associated to mass, momentum and energy densities. System (678) is an underdetermined system of $\mathrm{f}$ ve equations for the six primitive variables. In order to close the system, it is common to assume the flui obeys an ideal gas equation of state

$$
p=\rho_{0} e(\Gamma-1)
$$

with $\Gamma$ the adiabatic index. 
Let us now restrict to a spherically symmetric space-time described in spherical coordinates. The most general $3+1$ line element with these conditions is

$$
\begin{aligned}
d s^{2} & =\left(-\alpha^{2}+\beta_{r} \beta^{r}\right) d t^{2}+2 \beta_{r} d t d r \\
& +\gamma_{r r} d r^{2}+\gamma_{\theta \theta}\left(d \theta^{2}+\sin ^{2} \theta d \phi^{2}\right),
\end{aligned}
$$

where all the functions depend on $(t, r)$. From this metric one has that if $\gamma=\operatorname{det}\left(\gamma_{i j}\right)$, then $\sqrt{\gamma}=\sqrt{\gamma_{r r}} \gamma_{\theta \theta} \sin \theta$.

where the state variables, flu es and source vectors are explicitly

$$
\left.\begin{array}{rl}
\mathbf{u}= & \left(\begin{array}{c}
D \\
J_{r} \\
J_{\theta} \\
J_{\phi} \\
\tau
\end{array}\right)=\left(\begin{array}{c}
D \\
J_{r} \\
0 \\
0 \\
\tau
\end{array}\right), \\
\mathbf{F}^{r}(\mathbf{u})= & \left(\begin{array}{c}
\alpha\left(v^{r}-\frac{\beta^{r}}{\alpha}\right) D \\
\alpha\left(v^{r}-\frac{\beta^{r}}{\alpha}\right) J_{r}+\alpha \sqrt{\gamma} p \delta_{r}^{r} \\
\alpha\left(v^{r}-\frac{\beta^{r}}{\alpha}\right) J_{\theta}+\alpha \sqrt{\gamma} p \delta_{\theta}^{r} \\
\alpha\left(v^{r}-\frac{\beta^{r}}{\alpha}\right) J_{\phi}+\alpha \sqrt{\gamma} p \delta_{\phi}^{r} \\
\alpha\left(v^{r}-\frac{\beta^{r}}{\alpha}\right) \tau+\alpha \sqrt{\gamma} p v^{r}
\end{array}\right)=\left(\begin{array}{c}
\alpha\left(v^{r}-\frac{\beta^{r}}{\alpha}\right) D \\
\alpha\left(v^{r}-\frac{\beta^{r}}{\alpha}\right) J_{r}+\alpha \sqrt{\gamma} p \\
0 \\
\alpha\left(v^{\theta}-\frac{\beta^{\theta}}{\alpha}\right) D \\
\mathbf{F}^{\theta}(\mathbf{u})= \\
\alpha\left(v^{\theta}-\frac{\beta^{\theta}}{\alpha}\right) J_{r}+\alpha \sqrt{\gamma} p \delta_{r}^{\theta} \\
\alpha\left(v^{\theta}-\frac{\beta^{\theta}}{\alpha}\right) J_{\theta}+\alpha \sqrt{\gamma} p \delta_{\theta}^{\theta} \\
\alpha\left(v^{\theta}-\frac{\beta^{\theta}}{\alpha}\right) J_{\phi}+\alpha \sqrt{\gamma} p \delta_{\phi}^{\theta} \\
\alpha\left(v^{\theta}-\frac{\beta^{\theta}}{\alpha}\right) \tau+\alpha \sqrt{\gamma} p v^{\theta}
\end{array}\right) \\
\left.\mathbf{S}^{r}\right) \tau+\alpha \sqrt{\gamma} p v^{r}
\end{array}\right),
$$

Since $\mathbf{F}^{\theta}$ and $\mathbf{F}^{\phi}$ have each a non-zero component, it is worth discussing this their influenc on the equations (11). Before that, we write down the non-zero stress energy tensor components that will contribute to sources. Using equations (2) and (3) these components are

$$
\begin{aligned}
& T^{00}=\frac{1}{\alpha^{2}}\left(\rho_{0} h W^{2}-p\right), \quad T^{r 0}=\frac{\rho_{0} h W^{2}}{\alpha}\left(v^{r}-\frac{\beta^{r}}{\alpha}\right)+\frac{\beta^{r}}{\alpha^{2}} p \\
& T^{r r}=\rho_{0} h W^{2}\left(v^{r}-\frac{\beta^{r}}{\alpha}\right)^{2}+\left(\frac{1}{\gamma_{r r}}-\frac{\beta^{r} \beta^{r}}{\alpha^{2}}\right) p, \quad T^{\theta \theta}=\frac{p}{\gamma_{\theta \theta}}, \quad T^{\phi \phi}=\frac{p}{\gamma_{\phi \phi}} .
\end{aligned}
$$


The other components, $T^{0 \theta}, T^{0 \phi}, T^{r \theta}, T^{r \phi}, T^{\theta \phi}$, are zero either because $u^{\theta}=u^{\phi}=0$ or because $g^{0 \theta}=g^{0 \phi}=g^{r \theta}=$ $g^{r \phi}=g^{\theta \phi}=0$. The sources also involve the Christoffel symbols for metric (10), that we show explicitly in Appendix A.

With the non-zero stress-energy tensor components and the non-zero Christoffel symbols, the entries of the source vector in (12) are

$$
\begin{aligned}
& 0=0 \\
& \alpha \sqrt{\gamma} T^{\mu \nu} g_{\nu \sigma} \Gamma_{\mu r}^{\sigma}=\alpha \sqrt{\gamma}\left(T^{00}\left[\left(-\alpha^{2}+\gamma_{r r}\left(\beta^{r}\right)^{2}\right) \Gamma_{0 r}^{0}+\gamma_{r r} \beta^{r} \Gamma^{r}{ }_{0 r}\right]+T^{r 0}\left[\left(-\alpha^{2}+\gamma_{r r}\left(\beta^{r}\right)^{2}\right) \Gamma^{0}{ }_{r r}\right.\right. \\
& \left.\left.+\gamma_{r r} \Gamma_{0 r}^{r}+\gamma_{r r} \beta^{r}\left(\Gamma_{r r}^{r}+\Gamma_{0 r}^{0}\right)\right]+T^{r r}\left[\gamma_{r r} \Gamma_{r r}^{r}+\gamma_{r r} \beta^{r} \Gamma^{0}{ }_{r r}\right]+T^{\theta \theta} \gamma_{\theta \theta} \Gamma^{\theta}{ }_{\theta r}+T^{\phi \phi} \gamma_{\phi \phi} \Gamma_{\phi r}^{\phi}\right), \\
& \alpha \sqrt{\gamma} T^{\mu \nu} g_{\nu \sigma} \Gamma^{\sigma}{ }_{\mu \theta}=T^{\phi \phi} \gamma_{\phi \phi} \Gamma^{\phi}{ }_{\phi \theta}=\alpha \sqrt{\gamma} p \cot \theta \text {, } \\
& \alpha \sqrt{\gamma} T^{\mu \nu} g_{\nu \sigma} \Gamma_{\mu \phi}^{\sigma}=0, \\
& \alpha \sqrt{\gamma}\left(T^{\mu 0} \partial_{\mu} \alpha-\alpha T^{\mu \nu} \Gamma^{0}{ }_{\mu \nu}\right)=\alpha \sqrt{\gamma}\left(T^{00}\left(\partial_{0} \alpha-\alpha \Gamma^{0}{ }_{00}\right)\right. \\
& \left.+T^{r 0}\left(\partial_{r} \alpha-2 \alpha \Gamma_{r 0}^{0}\right)-\alpha\left(T^{r r} \Gamma^{0}{ }_{r r}+T^{\theta \theta} \Gamma_{\theta \theta}^{0}+T^{\phi \phi} \Gamma_{\phi \phi}^{0}\right)\right) .
\end{aligned}
$$

Now it is possible to study the effects of the non-trivial entries of $\mathbf{F}^{\theta}$ and $\mathbf{F}^{\phi}$ in (11/12). To study the third term $\partial \mathbf{F}^{\theta} / \partial \theta$ in Eq. (11), notice that the third entry of $\mathbf{F}^{\theta}$ in (12), contributes to the third term of the third equation in (11) because

$$
\begin{aligned}
\frac{\partial \mathbf{F}^{\theta}}{\partial \theta} & =\partial_{\theta}\left[\alpha(t, r) \sqrt{\gamma_{r r}(t, r)} \gamma_{\theta \theta}(t, r) \sin \theta p(t, r)\right] \\
& =\alpha(t, r) \sqrt{\gamma_{r r}(t, r)} \gamma_{\theta \theta}(t, r) \cos \theta p(t, r) .
\end{aligned}
$$

On the other hand, the third entry of the sources, namely the third of Eqs. (14) is precisely $\alpha \sqrt{\gamma_{r r}} \gamma_{\theta \theta} p \sin \theta \cot \theta$ and therefore these terms in the third of Eqs. (11) cancel out, which implies that the evolution equation for $J_{\theta}$ is an identity.

Let us now see the fourth entry of $\mathbf{F}^{\phi}$, notice that $\alpha \sqrt{\gamma} p=\alpha(t, r) \sqrt{\gamma_{r r}(t, r)} \gamma_{\theta \theta}(t, r) \sin \theta p(t, r)$, therefore $\partial \mathbf{F}^{\phi} / \partial \phi=0$, and thus the fourth term vanishes in (11) for all components of $\mathbf{F}^{\phi}$, and the evolution equation for $J_{\phi}$ becomes an identity as well.

With the non-trivial equations, one can defin an Initial Value Problem for the evolution of a perfect fluid with radial flux on top of a spherically symmetric space-time, written in the form (11), with three non-trivial equations only:

$$
\begin{aligned}
\partial_{t} D & +\partial_{r}\left(\alpha\left[v^{r}-\frac{\beta^{r}}{\alpha}\right] D\right)=0, \\
\partial_{t} J_{r} & +\partial_{r}\left(\alpha\left[v^{r}-\frac{\beta^{r}}{\alpha}\right] J_{r}+\alpha \sqrt{\gamma} p\right) \\
& =\alpha \sqrt{\gamma} T^{\mu \nu} g_{\nu \sigma} \Gamma^{\sigma}{ }_{\mu r}, \\
\partial_{t} \tau & +\partial_{r}\left(\alpha\left[v^{r}-\frac{\beta^{r}}{\alpha}\right] \tau+\alpha \sqrt{\gamma} p v^{r}\right) \\
& =\alpha \sqrt{\gamma}\left(T^{\mu 0} \partial_{\mu} \alpha-\alpha T^{\mu \nu} \Gamma_{\mu \nu}^{0}\right),
\end{aligned}
$$

where $D=D(t, r), J_{r}=J_{r}(t, r), \tau=\tau(t, r)$ depend on $\rho_{0}, v^{r}, p, e$, through Eqs. (8). The problem is define on the domain $\mathcal{D}=r \in\left[r_{\min }, r_{\text {max }}\right] \times t \in\left[0, t_{f}\right]$, with initial conditions $\rho_{0}(0, r), v^{r}(0, r), e(0, r), p(0, r)$ and boundary conditions $D\left(t,\left\{r_{\min }, r_{\max }\right\}\right), J_{r}\left(t,\left\{r_{\min }, r_{\max }\right\}\right)$, $\tau\left(t,\left\{r_{\min }, r_{\max }\right\}\right)$. This is a system of three equations for three conservative variables $D, J_{r}, \tau$, related to the four primitive variables $\rho_{0}, v^{r}, e, p$, which is closed using the $\operatorname{EoS}(9)$.

We solve this evolution problem using High Resolution Shock Capturing Methods (HRSC), which have become the standard method in Numerical Relativity with matter [6]. Here we present a brief summary of these methods and refer to the educative paper [7] for a detailed description. The reason to use these methods is that shocks can form due to the quasi-linear nature of Euler's equations, and Finite Volume based methods like HRSC methods help constructing weak solutions of problems with discontinuities.

\subsection{Summary of a simple HRSC implementation}

High Resolution Shock Capturing Methods are based on a Finite Volume discretization of an Initial Value Problem (IVP). In [7] a detailed description of these methods applied to the solution of relativistic flui equations can be found, which is based on the seminal descriptions in $[4,5,8]$.

Assume one has an IVP associated to a set of flu balance law equations, say in the $(t, r)$ domain

$$
\frac{\partial \mathbf{u}}{\partial t}+\frac{\partial(\mathbf{F}(\mathbf{u}))}{\partial r}=\mathbf{S}(\mathbf{u}),
$$

where $\mathbf{u}$ is a state vector, $\mathbf{F}$ a vector of flu es and $\mathbf{S}$ a vector with sources, like in our case (15). These equations can be solved using the method of lines, provided there are appropriate right hand sides for the evolution of $\mathbf{u}$ as described in Appendix B. An essential property of Euler equations, like ours in (15), is that even for smooth initial data, shocks can be formed due to the cross of characteristic lines, and consequently spatial derivatives of flu es become undefined This problem is solved using a Finite Volume approach that solves local Riemann Problems, by assuming the flui elements are define within cells, a method described in [6,9-11]. A Riemann problem is define at each boundary between two neighboring cells, one to the left $L$, one to the right $R$, in 
which average values of the variables and flu es are define (see [7]).

The implementation firs requires to rewrite the equations as

$$
\frac{\partial \mathbf{u}}{\partial t}+\frac{d \mathbf{F}}{d \mathbf{u}} \frac{\partial \mathbf{u}}{\partial r}=\mathbf{S}(\mathbf{u}),
$$

where $d \mathbf{F} / d \mathbf{u}$ is a Jacobian matrix whose eigenvalues are the local characteristic speeds of the characteristic modes of the system of equations. With the characteristic information, different approximate values of the original term $\partial(\mathbf{F}(\mathbf{u})) / \partial r$ in (16) can be constructed. For example Roe, HLLE, HLLC, HLLD approximate flu es, with different properties each, for example their capacity to track the fast modes only, or contact discontinuity modes, etcetera, provide approximate value of the flu es [6,9-11].

We assume the IVP associated with the evolution Eqs. (15) and (17), is define on the domain $\mathcal{D}=$ $\left[r_{\min }, r_{\max }\right] \times\left[0, t_{f}\right]$. The implementation requires the defini tion of a discrete domain $\mathcal{D}_{d}$, here we consider a uniform grid given by $\mathcal{D}_{d}=\left\{\left(t^{n}, r_{i}\right) \mid r_{i}=r_{\min }+i \Delta r, t^{n}=n \Delta t, i=\right.$ $\left.0, \ldots N_{r}\right\}$, with resolutions $\Delta r=\left(r_{\max }-r_{\min }\right) / N_{r}$ and $\Delta t=$ $C \Delta r$. A cell of the domain centered at $\left(t^{n+1 / 2}, x_{i+1 / 2}\right)$ is define by four vertices at $\left(t^{n}, r_{i}\right),\left(t^{n}, r_{i+1}\right),\left(t^{n+1}, r_{i}\right)$ and $\left(t^{n+1}, r_{i+1}\right)$.

Lines of constant $r_{i}$ become boundaries between neighboring cells $\left(t^{n+1 / 2}, x_{i-1 / 2}\right)$ at the left $L$ and $\left(t^{n+1 / 2}, x_{i+1 / 2}\right)$ at the right $R$. The flu es across these boundaries can be calculated using approximate Riemann solvers associated to formulas for the flu es at cells $L$ and $R[6,11]$.

As a particular case, here we use the HLLE approximate flu formula given by [11-13]

$$
\begin{aligned}
& \overline{\mathbf{F}}_{i+1 / 2}^{H L L E}= \\
& \frac{\lambda^{+} \mathbf{F}\left(\tilde{\mathbf{u}}_{i+1 / 2}^{L}\right)-\lambda^{-} \mathbf{F}\left(\tilde{\mathbf{u}}_{i+1 / 2}^{R}\right)+\lambda^{+} \lambda^{-}\left(\tilde{\mathbf{u}}_{i+1 / 2}^{R}-\tilde{\mathbf{u}}_{i+1 / 2}^{L}\right)}{\lambda^{+}-\lambda^{-}} .
\end{aligned}
$$

In our problem (15) we have three equations, and therefore a $3 \times 3$ Jacobian matrix in (17) with eigenvalues $\lambda_{1}, \lambda_{2}, \lambda_{3}$. In terms of these eigenvalues $\lambda^{+}$and $\lambda^{-}$are define by

$$
\begin{aligned}
& \lambda^{+}=\max \left(0, \lambda_{1}^{R}, \lambda_{2}^{R}, \lambda_{3}^{R}, \lambda_{1}^{L}, \lambda_{2}^{L}, \lambda_{3}^{L}\right), \\
& \lambda^{-}=\min \left(0, \lambda_{1}^{R}, \lambda_{2}^{R}, \lambda_{3}^{R}, \lambda_{1}^{L}, \lambda_{2}^{L}, \lambda_{3}^{L}\right),
\end{aligned}
$$

with $\lambda_{i}^{L}$ and $\lambda_{i}^{R}$ the eigenvalues at cells $L$ and $R$ of the Jacobian matrix in Eq. (17). The quantities $\mathbf{u}_{i+1 / 2}^{L}$ and $\mathbf{u}_{i+1 / 2}^{R}$ are the values of the state variables at the centers of the cell at the Left and at the Right, respectively, and $\overline{\mathbf{u}}_{i}=\int_{i-1 / 2}^{i+1 / 2} u(t, r) d r / \Delta r$. The values of these variables can be reconstructed in various ways, for instance the Godunov reconstruction assumes these variables are constant across the entire cell. Other reconstructors assume the variables are linear piece-wise using the information in nearest neighbor cells, for example the minmod method that we use here. These two methods are very well detailed in the educative paper [7]. There are other higher order reconstructors that involve the information from second nearest neighbors, that limit the slope of piece-wise variables, or the flu es $[6,11]$.

With an approximate flu formula like (18) one can calculate the evolution of the variables using the method of lines as explained in Appendix B with the appropriate right hand sides:

$$
\frac{\partial \overline{\mathbf{u}}}{\partial t}=-\frac{\left(\overline{\mathbf{F}}_{i+1 / 2}^{H L L E}-\overline{\mathbf{F}}_{i-1 / 2}^{H L L E}\right)}{\Delta x}+\overline{\mathbf{S}}_{i}^{n+1 / 2}
$$

which in principle allow the integration in time.

The steps we follow to construct the flu es are the following:

a) Reconstruct the variables $\mathbf{u}_{i+1 / 2}^{L}$ and $\mathbf{u}_{i+1 / 2}^{R}$ at $L$ and $R$ cells. Notice that in our problem (15) the flu es depend both on primitive and conservative variables, and therefore not only conservative, but primitive variables need to be reconstructed as well. This can be done using constant piece-wise or linear reconstructors, for example Godunov or minmod methods respectively $[6,7]$. We use minmod.

b) Calculate the eigenvalues $\lambda_{i}^{L}$ and $\lambda_{i}^{R}$ of the Jacobian matrix for the system (15).

c) Calculate $\lambda^{+}$and $\lambda^{-}$using (19).

d) Calculate the flu es $\mathbf{F}\left(\tilde{\mathbf{u}}_{i+1 / 2}^{L}\right)$ and $\mathbf{F}\left(\tilde{\mathbf{u}}_{i+1 / 2}^{R}\right)$.

e) Calculate the HLLE flu es with (18).

f) Use the method of lines with the expression (20) to integrate in time, as described in the Appendix B.

g) Implement boundary conditions on the conservative variables.

When applied to Euler equations, density must be finit in order to avoid divergencies, for example, the specifi enthalpy in (2) diverges for zero density, which will immediately translate the divergency to all other quantities of the equations. Then we need to set a minimum value of $\rho_{0}$ known as an atmosphere value $\rho_{a t m}$, because it acts like a background value in the whole numerical domain at all times.

The reader can enhance the accuracy of the HRSC method by using other flu formulae from the same family of methods, namely like HLLC, HLLD, which are nearly straightforward generalizations of the HLLE used here, and also experiment with other numerical flu constructions, like Roe and Marquina methods [6,11]. Another part that can be enhanced is the reconstruction of variables using higher order reconstructors [6]. 


\subsection{Implementation of HRSC methods for a spherically symmetric flow}

According to item (b), for the implementation of these HRSC methods one needs the characteristic structure of Eqs. (11). We do not calculate the eigenvalues here, instead we take them from Eqs. (21) and (22) in [5], which are

$$
\begin{aligned}
& \lambda_{1}=\alpha v^{r}-\beta^{r}, \\
& \lambda_{2}=\frac{\alpha}{1-v_{r} v^{r} c_{s}^{2}}\left[v^{r}\left(1-c_{s}^{2}\right)+c_{s} \sqrt{\left(1-v_{r} v^{r}\right)\left[\gamma^{r r}\left(1-v_{r} v^{r} c_{s}^{2}\right)-v^{r} v^{r}\left(1-c_{s}^{2}\right)\right]}\right]-\beta^{r}, \\
& \lambda_{5}=\frac{\alpha}{1-v_{r} v^{r} c_{s}^{2}}\left[v^{r}\left(1-c_{s}^{2}\right)-c_{s} \sqrt{\left(1-v_{r} v^{r}\right)\left[\gamma^{r r}\left(1-v_{r} v^{r} c_{s}^{2}\right)-v^{r} v^{r}\left(1-c_{s}^{2}\right)\right]}\right]-\beta^{r},
\end{aligned}
$$

with $c_{s}$ the speed of sound define as

$$
h c_{s}^{2}=\chi+\left(\frac{p}{\rho_{0}^{2}}\right) \kappa, \quad \chi=\frac{\partial p}{\partial \rho_{0}}, \quad+\kappa=\frac{\partial p}{\partial e} .
$$

For the $\operatorname{EoS} p=\rho_{0} e(\Gamma-1), \chi=\partial p / \partial \rho_{0}=e(\Gamma-1)=$ $p / \rho_{0}$ and $\kappa=\partial p / \partial e=\rho_{0}(\Gamma-1)$, therefore using that $h=1+e+p / \rho_{0}=1+\left(p / \rho_{0}\right)(\Gamma / \Gamma-1)$, the speed of sound is

$$
c_{s}^{2}=\frac{\chi+\kappa \frac{p}{\rho_{0}^{2}}}{h}=\frac{p(\Gamma-1) \Gamma}{\rho_{0}(\Gamma-1)+p \Gamma} .
$$

As mentioned in item (a) above, the flu es of system (15) are written in terms of primitive and conservative variables, which obliges one to calculate the primitive variables $\rho_{0}, v^{r}, p, e$ immediately after there are new values for the conservative variables $D, J_{r}, \tau$ during the evolution. The primitive variables in terms of conservative variables are

$$
\begin{aligned}
v^{r} & =\frac{J_{r}}{\sqrt{\gamma} \rho_{0} h W^{2} \gamma_{r r}}=\frac{J_{r}}{(\tau+\sqrt{\gamma} p+D) \gamma_{r r}}, \\
\rho_{0} & =\frac{D}{W \sqrt{\gamma}}=\frac{D}{\sqrt{\gamma}} \sqrt{1-\gamma_{r r}\left(v^{r}\right)^{2}}, \\
p & =p\left(\rho_{0}, e\right) .
\end{aligned}
$$

The pressure depends on density and internal energy through the ideal gas EoS. Developing the third of equations (24) we have

$$
\begin{aligned}
p & =\rho_{0} e(\Gamma-1) \\
& =(\Gamma-1)\left(\rho_{0} h-\rho_{0}-p\right) \\
& =(\Gamma-1)\left(\frac{1}{W^{2}}\left[\frac{\tau}{\sqrt{\gamma}}+p+\frac{D}{\sqrt{\gamma}}\right]-\rho_{0}-p\right) \\
& =\rho_{0}(\Gamma-1)\left(\frac{\tau+p \sqrt{\gamma}+D-\rho_{0} \sqrt{\gamma} W^{2}-p \sqrt{\gamma} W^{2}}{\rho_{0} \sqrt{\Gamma} W^{2}}\right) \\
& =\rho_{0}(\Gamma-1)\left(\frac{\tau+D[1-W]+p \sqrt{\gamma}\left[1-W^{2}\right]}{D W}\right), \quad(25)
\end{aligned}
$$

where the Lorentz factor

$$
\begin{aligned}
W & =\frac{1}{\sqrt{1-\gamma_{r r}\left(v^{r}\right)^{2}}}=\frac{1}{\sqrt{1-\frac{J_{r}^{2}}{\gamma_{r r}(\tau+\sqrt{\gamma} p+D)^{2}}}} \\
& =\frac{\sqrt{\gamma_{r r}}(\tau+\sqrt{\gamma} p+D)}{\sqrt{\gamma_{r r}(\tau+\sqrt{\gamma} p+D)^{2}-J_{r}^{2}}},
\end{aligned}
$$

has to be substituted into (25), which in turn has to be solved for $p$. The solution is commonly calculated using the Newton-Raphson method. For each cell of the numerical domain one has to fin the zeroes of the function

$$
f=p-\rho_{0}(\Gamma-1)\left(\frac{\tau+D[1-W]+p \sqrt{\gamma}\left[1-W^{2}\right]}{D W}\right),
$$

with

$$
W=\frac{\sqrt{\gamma_{r r}}(\tau+\sqrt{\gamma} p+D)}{\sqrt{\gamma_{r r}(\tau+\sqrt{\gamma} p+D)^{2}-J_{r}^{2}}} .
$$

For this we iterate the value of pressure

$$
p_{k}=p_{k-1}-\frac{f(p)}{f^{\prime}(p)}
$$

at each cell until a tolerance is achieved. An initial guess is needed to start the loop, for example for $k=0$ we defin $p_{k=0}=\left(p_{i}+p_{i+1}\right) / 2$, which is an average of the pressure at two neighboring cells from the previous time step of the evolution.

We stop the process once the following criterion, relating the difference between consecutive values of the pressure weighed with the average between the two values is fulfille

$$
\left|\frac{p_{k}-p_{k-1}}{\frac{1}{2}\left(p_{k}+p_{k-1}\right)}\right|<10^{-12}
$$

a criterion achieved after less than ten iterations for each cell for the examples in this paper. Finally, once the pressure has been calculated, the internal energy is obtained from the equation of state $e=p /(\Gamma-1) \rho_{0}$, which is finit as long as $\rho_{0} \neq 0$, which is the case due to the atmosphere. With this, all primitive variables are now known. With them it is possible to calculate the eigenvalues $\lambda_{i}$ with (21) needed in item (c), then the flu es in (d).

\section{Test fluid case}

Now we have all the matter related ingredients needed for the simulation of the accretion process, we only need to specify 
the space-time. In this firs scenario, the flui evolves on top of the fi ed space-time background of a Schwarzschild black hole, that we describe in Eddington-Finkelstein (EF) coordinates, whose line element in spherical coordinates is

$$
\begin{aligned}
d s^{2} & =-\left(1-\frac{2 M}{r}\right) d t^{2}+\frac{4 M}{r} d t d r \\
& +\left(1+\frac{2 M}{r}\right) d r^{2}+r^{2}\left(d \theta^{2}+\sin ^{2} \theta d \phi^{2}\right),
\end{aligned}
$$

with $M$ the mass of the black hole. The reason for writing the metric using EF coordinates is because these coordinates penetrate the event horizon as opposed to the Schwarzschild coordinates that diverge at the horizon as detailed below.

From this line element one can extract the geometrical quantities needed to write down all the elements of the evolution equations for the fluid Identifying eq. (10) with (27) one obtains the $3+1$ gauge and metric functions

$$
\begin{aligned}
\alpha & =\frac{1}{\sqrt{1+\frac{2 M}{r}}}, \\
\beta^{i} & =\left(\beta^{r}, 0,0\right)=\left(\frac{2 M}{r} \frac{1}{1+\frac{2 M}{r}}, 0,0\right), \\
\gamma_{i j} & =\left(\gamma_{r r}, \gamma_{\theta \theta}, \sin ^{2} \theta \gamma_{\theta \theta}\right)=\operatorname{diag}\left(1+\frac{2 M}{r}, r^{2}, r^{2} \sin ^{2} \theta\right), \\
\beta_{r} & =\gamma_{r r} \beta^{r}=\frac{2 M}{r}, \\
\sqrt{\gamma} & =\sqrt{\gamma_{r r} \gamma_{\theta \theta} \gamma_{\phi \phi}}=r^{2} \sin \theta \sqrt{1+\frac{2 M}{r}},
\end{aligned}
$$

which are needed to calculate the stress energy tensor components (13), sources (14), flu es (18) and the equation for the pressure (25).

Additionally, the sources involve Christoffel symbols, and here we write down the non-zero ones for the EF spacetime:

$$
\begin{array}{ll}
\Gamma_{00}^{0}=\frac{2 M^{2}}{r^{3}}, & \Gamma_{0 r}^{0}=\frac{M}{r^{2}}\left(1+\frac{2 M}{r}\right), \\
\Gamma_{0 r}^{r}=-\frac{2 M^{2}}{r^{3}}, & \Gamma_{r r}^{0}=\frac{2 M}{r^{2}}\left(1+\frac{M}{r}\right), \\
\Gamma_{r r}^{r}=-\frac{M}{r^{2}}\left(1+\frac{2 M}{r}\right), & \Gamma^{\theta}{ }_{r \theta}=\frac{1}{r}, \\
\Gamma_{r \phi}^{\phi}=\frac{1}{r}, & \Gamma_{\theta \theta}^{0}=-2 M, \\
\Gamma_{\phi \phi}^{0}=-2 M \sin ^{2} \theta, & \Gamma^{r}{ }_{00}=\frac{M}{r^{2}}\left(1-\frac{2 M}{r}\right), \\
\Gamma_{\theta \theta}^{r}=2 M-r, & \Gamma_{\phi \phi}^{r}=(2 M-r) \sin ^{2} \theta, \\
\Gamma_{\phi \phi}^{\theta}=-\sin \theta \cos \theta, & \Gamma_{\theta \phi}^{\phi}=\cot \theta .
\end{array}
$$

This completes the information needed to solve the evolution of a perfect flui being accreted by a black hole.
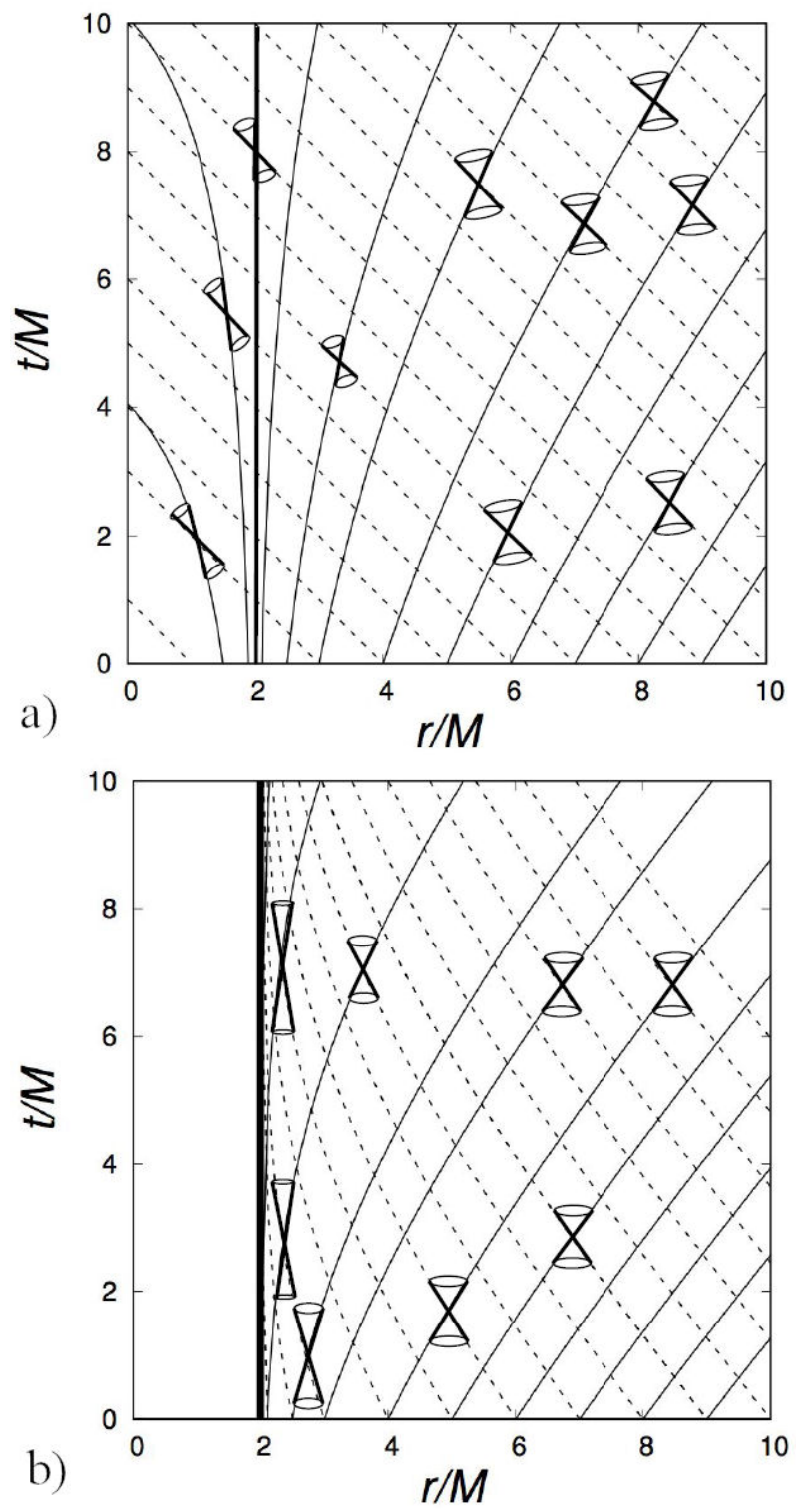

FIGURE 1. a) We show a bundle of null rays that illustrates the light cone structure of the Schwarzschild black hole space-time described with Eddington-Finkelstein coordinates. Continuous and dotted lines correspond to outgoing and ingoing null rays respectively. We draw a few light cones, to indicate the causal structure at different points of the domain. b) For comparison we also show the null ray structure in Schwarzschild coordinates outside the horizon, which shows how light cones collapse when $r$ approaches $2 M$.

\subsection{Numerical solution}

The numerical solution is a solution of a discrete version of the IVP in (15), which needs a well define domain, initial conditions and boundary conditions.

The physical problem will be the accretion of a flui initially with uniform density and velocity, which is a particular solution of the IVP (15). In what follows we present a description of details needed to achieve a successful accretion solution. 
It is a common practice to use geometric units $G=c=1$ for the construction of numerical solutions. In these units, as described in Appendix $\mathrm{C}$, length and time are in units of $M$. Therefore the domain will be define in these units.

We set the numerical domain such that the inner boundary is at $r_{\min }=M$, within the black hole horizon, in order to allow the flui to enter the horizon. The external boundary ideally has to be located at spatial infinit, which can be achieved with a compactifie radial coordinate (see, e.g., [14]). More commonly, the external boundary can be set far from the horizon using a logarithmic radial coordinate [15], or other sorts of stretched coordinates (e.g., [16]). In general, there is no prescription for a location of the external boundary, but it is always desirable to defin it as far away from the strong fiel region as possible, so that there the space-time is a small perturbation of Minkowski space-time. For simplicity we use a uniformly discretized numerical domain and set the external boundary at $r_{\max }=51 \mathrm{M}$, where the metric departs from that of Minkowski space-time by $4 \%$. The fina time is set to $t_{f}=1000 M$ in order for the accretion to get stationary.

Numerical Domain. We use the discrete domain $\mathcal{D}_{d}$ define above and we rewrite it here, $\mathcal{D}_{d}=\left\{\left(t^{n}, r_{i}\right) \mid r_{i}=\right.$ $\left.r_{\text {min }}+i \Delta r, t^{n}=n \Delta t, i=0, \ldots N_{r}, n=0,1,2, \ldots, N_{t}\right\}$, with resolutions $\Delta r=\left(r_{\max }-r_{\min }\right) / N_{r}$ and $\Delta t=C \Delta r$. We set $C=0.25$ and our base resolution to $\Delta r=0.05$ or equivalently $N_{r}=1000$.

Atmosphere. As mentioned before, the numerical method requires the density to be non-zero in order to avoid divergencies. We set the minimum rest mass density value to $\rho_{\text {atm }}=10^{-13}$, which is a value near the round-off error. That is, we set $\rho_{0}=\max \left(\rho_{0}, \rho_{\text {atm }}\right)$ when updating the rest mass density. In the examples below, the atmosphere value is orders of magnitude smaller that the maximum of the density. We can use such small value in our problem because no depletion regions are formed during the evolution. In full three dimensions the complex fl ws usually trigger the formation of depletion zones, where density and pressure decrease, and it is common to use bigger values of $\rho_{\text {atm }}$ in those scenarios.

Initial conditions. We consider the flui to have initially a constant density $\rho_{0 \text {,ini }}$ and constant inward velocity $v_{\text {ini }}^{r}$.

Boundary conditions. At the outer boundary in $r=r_{\max }$ we want to maintain the flu injected constantly. This is done by setting the right hand sides of $D, J_{r}, \tau$ of Eqs. (15) to zero at this boundary.

At the inner boundary $r=r_{\min }$, it is useful to see the light cone structure of the space-time. In EF coordinates the null rays obey the equation $d t / d r=(2 M \pm r) /(r-2 M)$, where the minus/plus sign stands for ingoing/outgoing rays, and all ingoing null rays have slope -1 . Then, null ray trajectories in the space-time $r t$-plane are given by $t=-r+r_{0}$ for ingoing rays and $t=r-r_{0}+4 M \ln \left(r-2 M / r_{0}-2 M\right)$ for outgoing ones, with $r_{0}$ the initial position of a particular ray at $t=0$. A bundle of these null rays is shown in Fig. 1. For comparison we also show the null rays for the space-time in Schwarzschild coordinates $d s^{2}=-(1-[2 M / r]) d t^{2}+$ $d r^{2} /(1-[2 M / r])+r^{2}\left(d \theta^{2}+\sin ^{2} \theta d \phi^{2}\right)$. Null rays for this metric obey the equation $d t / d r= \pm 1 / 1-(2 M / r)$, with solutions $t= \pm\left(r-r_{0}+2 M \ln \left((r-2 M) /\left(r_{0}-2 M\right)\right)\right)$. We show sample of these rays also in Fig. 1 for comparison.

What we want to stress is that in EF coordinates light cones remain open for all $r>0$, whereas in Schwarzschild coordinates the cones collapse when $r \rightarrow 2 M^{+}$, because there $d t / d x$ diverges. This property of EF coordinates allows one to consider a numerical domain that includes part of the region inside the event horizon, and set the inner boundary within this surface.

Moreover, in EF coordinates, due to the light cone structure, the information is expected to travel only toward the origin of coordinates for $r<2 M$ and not to escape from the horizon, as can be seen in Fig. 1. Then, an extrapolation of the values of the conservative variables suffice and no boundary condition is needed. Using the Lagrange interpolation formula with three points we set the values of conservative variables at $r_{\min }=r_{0}$ :

$$
\begin{aligned}
D_{0} & =3 D_{1}-3 D_{2}+D_{3}, \\
J_{0} & =3 J_{1}-3 J_{2}+J_{3}, \\
\tau_{0} & =3 \tau_{1}-3 \tau_{2}+\tau_{3} .
\end{aligned}
$$

Finally, with these initial and boundary conditions, the flui will evolve and acquire a non-uniform density, velocity and pressure profiles

Fortunately there are solutions to compare with in order to know whether the results are correct or not. Comparison with exact solutions at this stage is very important, because we will know whether the numerical solutions are correct.

Solutions for the radial stationary infall of a perfect flui toward a point-like accretor were firs constructed in Newtonian gravity by Bondi [17], and later on the solutions of stationary radial fl w on Schwarzschild's space-time were well established by Michel [18], that we consider here. These solutions contain two important cases, the pressure-less or dust and the ideal gas or Michel case that we present next.

The solution for dust corresponds to the accretion of a pressure-less perfect flui $p=0$ onto a Black Hole. The solution for the density and velocity profiles for the metric written in EF coordinates, was developed in [19], and reads as follows

$$
\begin{aligned}
& \rho_{\text {dust }}(r)=\frac{-C_{1}}{r^{2} \sqrt{\frac{2 M}{r}}}, \\
& v_{\text {dust }}^{r}(r)=-\frac{1}{\sqrt{1+\frac{r}{2 M}}\left(1+\sqrt{\frac{2 M}{r}}+\frac{2 M}{r}\right)},
\end{aligned}
$$

where $C_{1}$ is a constant of integration.

As a firs test we use (31) as initial conditions with $C=-0.01$, and fin that density and velocity profile remain time-independent, which shows that the exact solution is stable at least under numerical perturbations due to the numerical methods. 


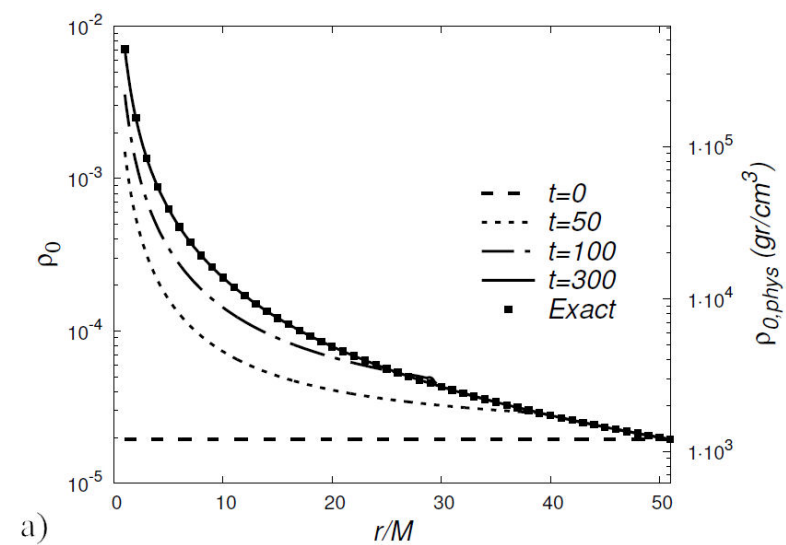

a)
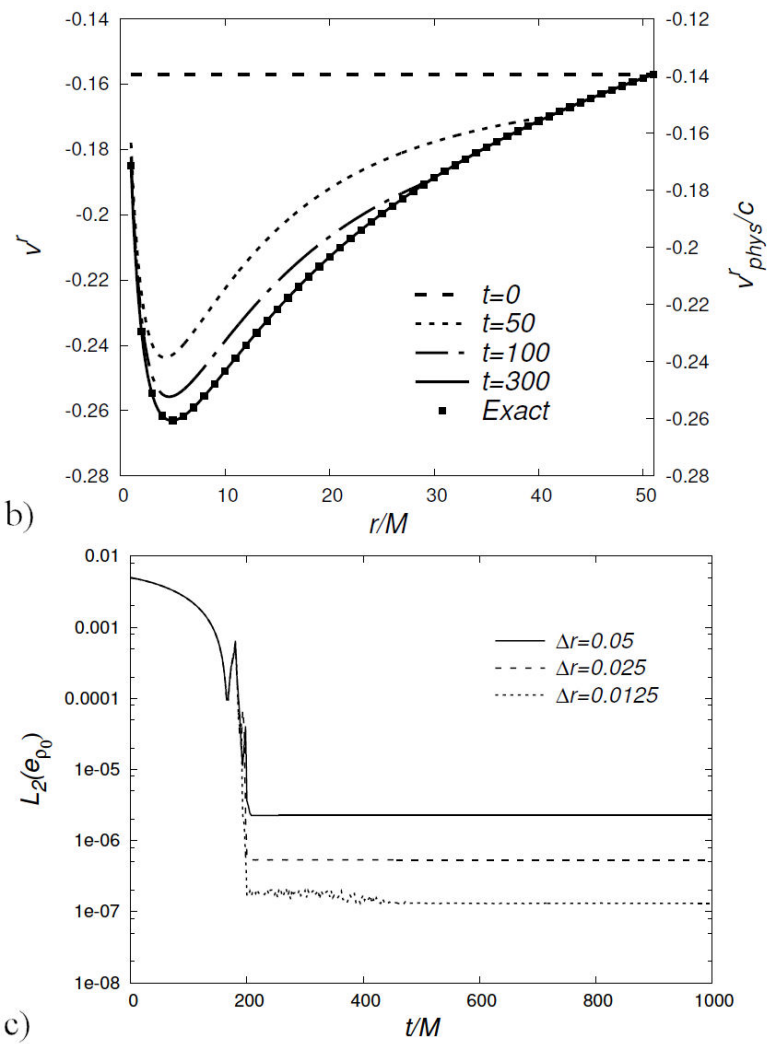

FIGURE 2. Numerical solution for $\rho_{0}$ and $v^{r}$, with $p=0$ and $C_{1}=-0.01$ at various times. Both, density and velocity start being constant and with time they tend toward the exact dust solution (31) in an attractor-like fashion. On the right axes the values in cgs units of density and velocity are shown for a black hole of mass $M=10^{6} M_{\odot}$, using the conversion factors in Appendix $\mathrm{C}$. The time of snapshots in the plots are in units of $M$, in physical units they correspond to $t=0,246.25,492.5,1477.5 \mathrm{~s}$. Finally in the last plot we show the convergence of the $L_{2}$ norm of the error in $\rho_{0}$ of the numerical solution.

For a non-trivial test, we start the evolution by setting the constant density to the constant value $\rho_{\text {ini }}=\rho_{\text {dust }}\left(r_{\max }\right)$ and $v_{i n i}^{r}=v_{\text {dust }}^{r}\left(r_{\max }\right)$. We solve the equations for $C_{1}=-0.01$ and the results are in Fig. 2. Both $\rho_{0}(r)$ and $v^{r}(r)$ start as constants and with time they change until the fl $\mathrm{w}$ becomes stationary. An interesting result is that the stationary solution approaches the exact dust solution (31) during the evolution, in an attractor-like fashion. In the last plot of Fig. 2 we show the $L_{2}$ norm of the error of the numerical solution with respect to the exact solution. The curves indicate firs that the numerical solution starts far from the exact solution and with time the error decreases by $t \sim 200$ to its minimum. It also shows how the error decreases as function of resolution and the convergence to the exact solution, a concept that will be clarifie in detail in Sec. 4.4.1.

An important technical point is in turn when solving problems with a pressure-less fluid When recovering the pressure in terms of conservative values using Eqs. $(25,26)$, one simply sets $p=0$, and afterwards the internal energy becomes $e=p /(\Gamma-1) / \rho_{0}$, which is zero for $\rho_{0}=$ $\max \left(\rho_{0}, \rho_{\text {atm }}\right)>0$.

Ideal gas. This is the spherically symmetric stationary infl $\mathrm{w}$ solution of a perfect fluid this time with pressure, onto a Black Hole [18].

Although the ideal gas solution is stationary like the dust case, it does not have a closed formula and the solution is numerical. We do not develop here the solution, although some didactic descriptions of this solution on the EF space-time are found in [19], the appendix of [20] and [21], indicating the steps to construct the density, pressure and velocity fiel of the solution, which is parameterized by critical density and radius $\rho_{c}$ and $r_{c}$, that fi all the flui variables.

In our example we use a relativistic flui with adiabatic index $\Gamma=4 / 3$. We set initial constant density and velocity profile to the values $\rho_{\text {ini }}=\rho_{0 \text {,ideal gas }}\left(r_{\max }\right)$ and $v_{\text {ini }}^{r}=$ $v_{\text {ideal gas }}^{r}\left(r_{\max }\right)$, using $\rho_{c}=0.1$ and $r_{c}=100$ in terms of the notation of [19-21]. Notice that we assume density and velocity profile to be constant initially. The flui will be constantly injected until the matter evolves in time approaching a stationary state.

We show the numerical solution of this problem in Fig. 3. Once again, density and velocity profile have initially constant profiles and with time they approach the exact solution. This exercise shows that the stationary solution behaves as an attractor in time. In fact, it would be possible to look for a basin of attraction by exploring various initial density and velocity profiles as well as measure the Lyapunov exponent of $L_{2}\left(e_{\rho_{0}}\right)$, indicating how the numerical solution approaches the exact solution as function of time.

In the last plot of Fig. 3 we show how the $L_{2}$ norm of the error in $\rho_{0}$ decreases with time until $t \sim 500$, when the difference stabilizes. This indicates that the numerical solution approaches the exact solution in time. We show this result for three different resolutions in order to show that the numerical solution converges with resolution, to the exact solution with second order, consistently with the accuracy order of the numerical methods used to construct the numerical solution, a concept that will be detailed in Sec. IV-D-1.

\subsection{Accretion rate}

One of the most important quantities in accretion processes is the mass accretion rate. Due to spherical symmetry the ac- 
a)

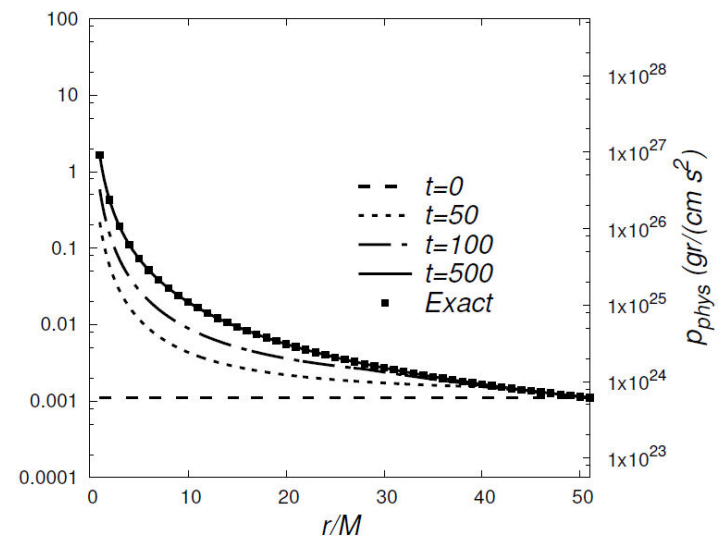

b)
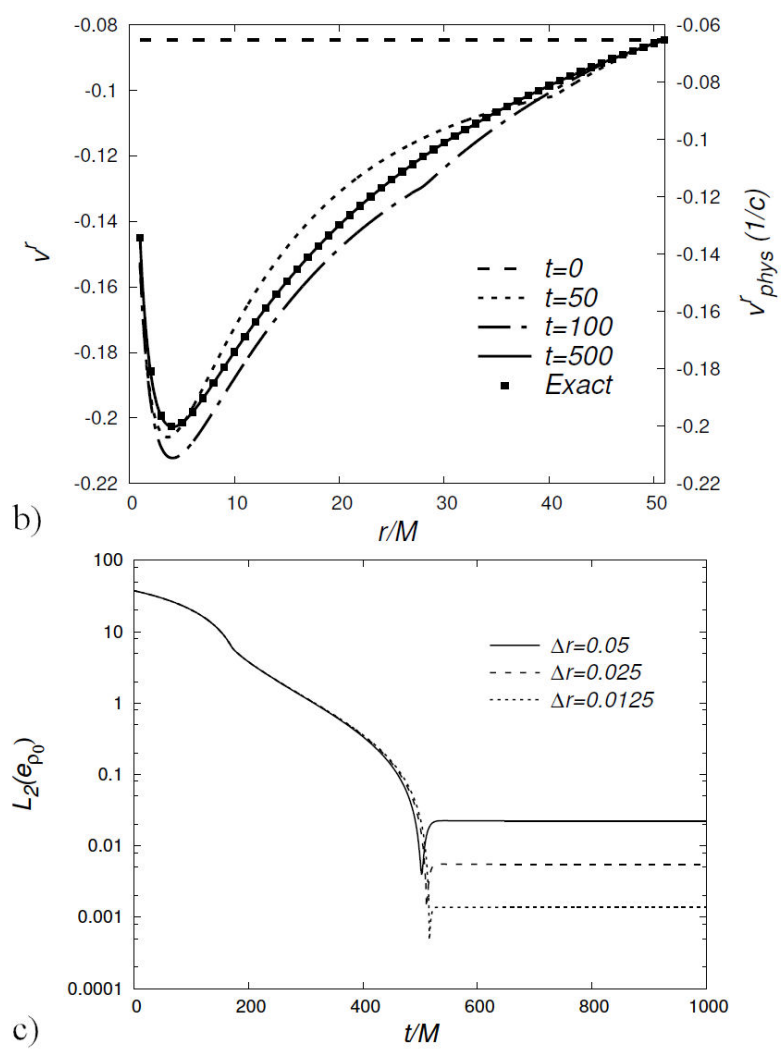

FIGURE 3. Numerical solution of $\rho_{0}, p$ and $v^{r}$. Notice that the numerical solution converges toward the exact solution with time. On the right axes the values in cgs units of density and velocity are shown for a black hole of mass $M=10^{6} M_{\odot}$, using the conversion factors in Appendix C. Finally in the last plot we show the convergence of the numerical solution toward the exact solution as function of resolution.

cretion mass rate is the integral of $\mathbf{F}_{1}^{r}$ in (12) on a spherical surface of radius $r_{d}$. For the Eddington-Finkelstein metric it reads

$$
\dot{M}_{a c c}\left(r_{d}\right)=\left.\int_{S^{2}\left(r_{d}\right)} \alpha\left(v^{r}-\frac{\beta^{r}}{\alpha}\right) D d S\right|_{r_{d}}
$$
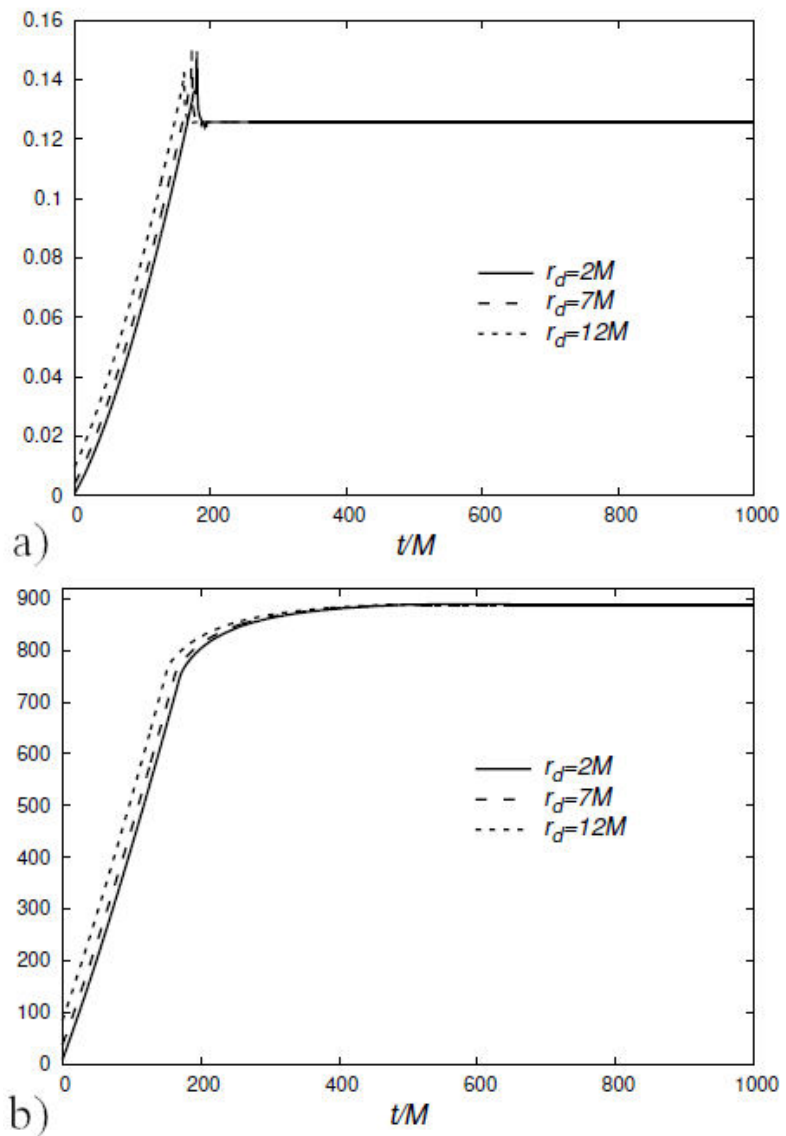

FIGURE 4. Accretion mass rate $\dot{M}_{a c c}$ for the cases of dust a) and ideal gas b), measured by detectors located at $r_{d}=2 M, 7 M, 12 M$, for the simulations in Figs. 2 and 3. The resolution used in this examples is $\Delta r=0.0125$.

$$
\begin{aligned}
& =\left.\int_{S^{2}\left(r_{d}\right)} \alpha\left(v^{r}-\frac{\beta^{r}}{\alpha}\right) \rho_{0} W \sqrt{\gamma} d \theta d \phi\right|_{r_{d}} \\
& =\left.4 \pi \alpha\left(v^{r}-\frac{\beta^{r}}{\alpha}\right) \rho_{0} W \sqrt{\gamma_{r r}} \gamma_{\theta \theta}\right|_{r_{d}},
\end{aligned}
$$

where all the factors in the expression are evaluated at $r=r_{d}$. The geometric factors correspond to those of the background EF space-time explicit in (28). This quantity is measured at various spherical surfaces of radius $r_{d}$ and plotted as a function of time in Fig. 4.

Notice that in the two cases the accretion rate starts growing and afterwards tends to a constant value asymptotically in time. Strictly speaking, the atmosphere intrinsic to the numerical method will also contribute all the way with a parasite accretion.

\section{The non-linear case}

In this second scenario, Einstein equations (EEs) are solved simultaneously with Euler equations for the fluid EEs 
can be cast in a variety of forms including the $3+1$, conformal, characteristic and harmonic formalisms. Since we have used the $3+1$ decomposition of the space-time for the description of flui dynamics equations, it is natural to use the $3+1$ decomposition of space-time for its own description. Within the $3+1$ decomposition approach, there are still a number of formulations of EEs, including for example the Arnowitt-Desser-Misner (ADM), BaumgarteShapiro-Shibata-Nakamura (BSSN), Bona-Masso, KidderScheel-Teukolsky (KST) formulations, among others (see e.g. $[6,22,23])$. The ADM is the simplest one, on top of which the other alternatives can be derived with desirable properties. Among the differences of $3+1$ formulations is the degree of hyperbolicity, which is related to the well posedness of an Initial Value Problem, which in turn is related to the stability of solutions $[22,23]$. The ADM formulation for instance is weakly hyperbolic, whereas BSSN and KST are strongly hyperbolic (see [24] for an educative example using the KST formulation). Concerning popularity, the BSSN formulation is the most commonly used for state of the art evolution of space-times involving black hole binaries that source Gravitational Waves $[25,26]$. In this academic paper though, we use the ADM formulation because one can implement straightforwardly the evolution equations, and because for the accretion processes here no instabilities emerge, we let the implementation of the accretion using the BSSN formulation for a follow-up work [27].

It is important to understand that these formulations defin evolution equations for geometric quantities, associated to an IVP, subject to the satisfaction of a set of constraint equations at initial time. The solution of this IVP is a chunk of the space-time itself, commonly define in a finit spatial domain and time. The chunk of space-time depends on the problem one is interested in, for example, the collision of two black holes might need a considerably big domain in time for the system to orbit, collide and relax, and a sufficientl big spatial domain for the black holes to have room for various orbits and the extraction of gravitational wave detectors far away from the holes.

The ADM equations for the $3+1$ metric (1) are explicitly twelve evolution equations and four constraints. These are six evolution equations for the components of the 3-metric $\gamma_{i j}$ of $\Sigma_{t}($ see $[22,23])$

$$
\partial_{t} \gamma_{i j}=-2 \alpha K_{i j}+D_{i} \beta_{j}+D_{j} \beta_{i}
$$

where $D_{i}$ is the covariant derivative on the hypersurface $\Sigma_{t}$ consistent with $\gamma_{i j}$. Six evolution equations for the components of the extrinsic curvature of $\Sigma_{t}$ on the ambient spacetime

$$
\begin{aligned}
\partial_{t} K_{i j} & =\alpha\left(R_{i j}-2 K_{i k} K_{j}^{k}+K K_{i j}\right)-D_{i} D_{j} \alpha \\
& -8 \pi \alpha\left(S_{i j}-\frac{1}{2} \gamma_{i j}(S-\rho)\right) \\
& +\beta^{k} \partial_{k} K_{i j}+K_{i k} \partial_{j} \beta^{k}+K_{k j} \partial_{i} \beta^{k},
\end{aligned}
$$

and the four constraints

$$
\begin{gathered}
R+K^{2}-K_{i j} K^{i j}=16 \pi \rho, \\
D_{j}\left(K^{i j}-\gamma^{i j} K\right)=8 \pi S^{i},
\end{gathered}
$$

respectively the Hamiltonian and three Momentum constraints.

Evolution and constraint Eqs. (33 34 35 36) involve the following geometric quantities of the hypersurfaces $\Sigma_{t}$, Ricci scalar $R$, extrinsic curvature components $K_{i j}$, and its trace $K$.

In these equations, matter terms are define as projections of the stress energy tensor along hypersurfaces $\Sigma_{t}$, their normal vectors $n^{\mu}$ or mixed projections $[22,23]$ :

$$
\begin{aligned}
\rho & =n_{\mu} n_{\nu} T^{\mu \nu}, \\
S^{i} & =-\gamma^{i j} n^{\mu} T_{\mu j}, \\
S_{i j} & =\gamma_{i \mu} \gamma_{j \nu} T^{\mu \nu}, \\
S & =\gamma^{i j} S_{i j},
\end{aligned}
$$

with $n_{\mu}=(-\alpha, 0,0,0)$ as we have set before in this paper. For the stress energy tensor of a perfect flui (2) with flui element velocities (3) these matter quantities read

$$
\begin{aligned}
\rho & =\rho_{0} h W^{2}-p, \\
S^{i} & =\rho_{0} h W^{2} v^{i}, \\
S_{i j} & =\rho_{0} h W^{2} v_{i} v_{j}+\gamma_{i j} p, \\
S & =\rho_{0} h W^{2} v_{i} v^{i}+3 p .
\end{aligned}
$$

Notice that $\rho$ has to be distinguished from the rest mass density $\rho_{0}$.

\subsection{Spherical symmetry}

As we have seen, for a spherically symmetric space-time written in spherical coordinates, the most general line element is (10), from which the components of the 3-metric of $\Sigma_{t}$ are $\gamma_{i j}=\operatorname{diag}\left(\gamma_{r r}, \gamma_{\theta \theta}, \sin ^{2} \theta \gamma_{\theta \theta}\right)$.

For the implementation of the numerical solution it is necessary specify all the terms in the constraint and evolution Eqs. (33-36). From (33) one find that the extrinsic curvature is diagonal $K_{i j}=\operatorname{diag}\left(K_{r r}, K_{\theta \theta}, \sin ^{2} \theta K_{\theta \theta}\right)$. In the evolution equations and constraints the following expression related to the extrinsic curvature are needed

$$
\begin{gathered}
K=\gamma^{i j} K_{i j}=\frac{K_{r r}}{\gamma_{r r}}+2 \frac{K_{\theta \theta}}{\gamma_{\theta \theta}}, \\
K_{i j} K^{i j}=K_{i j} \gamma^{i k} \gamma^{j l} K_{k l}=\frac{K_{r r}^{2}}{\gamma_{r r}^{2}}+2 \frac{K_{\theta \theta}^{2}}{\gamma_{\theta \theta}^{2}} .
\end{gathered}
$$

Matter. Considering only radial velocity $v^{i}=\left(v^{r}, 0,0\right)$, one find that the non-zero $3+1$ matter quantities $(38)$ are 


$$
\begin{aligned}
\rho & =\rho_{0} h W^{2}-p, \quad S^{r}=\rho_{0} h W^{2} v^{r}, \\
S_{r r} & =\rho_{0} h W^{2} v_{r} v_{r}+\gamma_{r r} p, \quad S_{\theta \theta}=\gamma_{\theta \theta}, \\
S_{\phi \phi} & =\sin ^{2} \theta S_{\theta \theta}, \quad S=\rho_{0} h W^{2} v_{r} v^{r}+3 p .
\end{aligned}
$$

Since we assume radial fl ws, $S^{\theta}=S^{\phi}=0$ and the only non-zero component of $S^{i}$ is $S^{r}=\rho_{0} h W^{2} v^{r}$. Also the non-diagonal components of $S_{i j}$ vanish.

The evolution equations (33 34) for the independent metric and curvature components $\gamma_{r r}, \gamma_{\theta \theta}, K_{r r}$ and $K_{\theta \theta}$ are finall

$$
\begin{aligned}
\partial_{t} \gamma_{r r} & =-2 \alpha K_{r r}+2 D_{r} \beta_{r} \\
& =-2 \alpha K_{r r}+2 \gamma_{r r} \partial_{r} \beta^{r}+\beta^{r} \partial_{r} \gamma_{r r} \\
\partial_{t} \gamma_{\theta \theta} & =-2 \alpha K_{\theta \theta}+2 D_{\theta} \beta_{\theta} \\
& =-2 \alpha K_{\theta \theta}+\beta^{r} \partial_{r} \gamma_{\theta \theta}, \\
\partial_{t} K_{r r} & =\alpha\left(R_{r r}-2 K_{r k} K_{r}^{k}+K K_{r r}\right)-D_{r} D_{r} \alpha \\
& +\beta^{k} \partial_{k} K_{r r}+K_{r k} \partial_{r} \beta^{k}+K_{k r} \partial_{r} \beta^{k} \\
& -8 \pi \alpha\left(S_{r r}-\frac{1}{2} \gamma_{r r}(S-\rho)\right) \\
& =\alpha\left(-\frac{\partial_{r r} \gamma_{\theta \theta}}{\gamma_{\theta \theta}}+\frac{1}{2} \frac{\left(\partial_{r} \gamma_{\theta \theta}\right)^{2}}{\gamma_{\theta \theta}^{2}}+\frac{1}{2} \frac{\left(\partial_{r} \gamma_{r r}\right)\left(\partial_{r} \gamma_{\theta \theta}\right)}{\gamma_{r r} \gamma_{\theta \theta}}\right. \\
& \left.-\frac{K_{r r}^{2}}{\gamma_{r r}}+2 \frac{K_{r r} K_{\theta \theta}}{\gamma_{\theta \theta}}\right)-\partial_{r r} \alpha+\frac{1}{2} \partial_{r} \alpha \frac{\partial_{r} \gamma_{r r}}{\gamma_{r r}} \\
& +\beta^{r} \partial_{r} K_{r r}+2 K_{r r} \partial_{r} \beta^{r}-8 \pi \alpha \\
& \times\left(S_{r r}-\frac{1}{2} \gamma_{r r}(S-\rho)\right), \\
\partial_{t} K_{\theta \theta} & =\alpha\left(R_{\theta \theta}-2 K_{\theta k} K_{\theta}^{k}+K K_{\theta \theta}\right)-D_{\theta} D_{\theta} \alpha+\beta^{k} \partial_{k} K_{\theta \theta} \\
& -8 \pi \alpha\left(S_{\theta \theta}-\frac{1}{2} \gamma_{\theta \theta}(S-\rho)\right)=\alpha\left(-\frac{1}{2} \frac{\partial_{r r} \gamma_{\theta \theta}}{\gamma_{r r}}\right. \\
+ & \left.1+\frac{1}{4} \frac{\left(\partial_{r} \gamma_{r r}\right)\left(\partial_{r} \gamma_{\theta \theta}\right)}{\gamma_{r r}^{2}}+\frac{K_{r r} K_{\theta \theta}}{\gamma_{r r}}\right)-\frac{1}{2} \frac{\partial_{r} \gamma_{\theta \theta}}{\gamma_{r r}} \partial_{r} \alpha \\
+ & \beta^{r} \partial_{r} K_{\theta \theta}-8 \pi \alpha\left(S_{\theta \theta}-\frac{1}{2} \gamma_{\theta \theta}(S-\rho)\right)
\end{aligned}
$$

where we have used the following expressions in the evolution equations (33) and (34):

$$
\begin{aligned}
D_{r} D_{r} \alpha & =\partial_{r r} \alpha-\Gamma_{r r}^{r} \partial_{r} \alpha=\partial_{r r} \alpha-\frac{1}{2} \frac{\partial_{r} \gamma_{r r}}{\gamma_{r r}} \partial_{r} \alpha, \\
D_{\theta} D_{\theta} \alpha & =-\Gamma_{\theta \theta}^{r} \partial_{r} \alpha=\frac{1}{2} \frac{\partial_{r} \gamma_{\theta \theta}}{\gamma_{r r}} \partial_{r} \alpha, \\
D_{r} \beta^{r} & =\partial_{r} \beta_{r}-\Gamma_{r r}^{r} \beta_{r}=\partial_{r} \beta_{r}-\frac{1}{2} \beta^{r} \partial_{r} \gamma_{r r}, \\
D_{\theta} \beta_{\theta} & =-\Gamma_{\theta \theta}^{r} \beta_{r}=\frac{1}{2} \beta^{r} \partial_{r} \gamma_{\theta \theta} .
\end{aligned}
$$

These covariant derivatives use the Christoffel symbols on $\Sigma_{t}$, being the non-zero ones

$$
\begin{array}{ll}
\Gamma_{r r}^{r}=\frac{1}{2} \frac{\partial_{r} \gamma_{r r}}{\gamma_{r r}}, & \Gamma_{\theta \theta}^{r}=-\frac{1}{2} \frac{\partial_{r} \gamma_{\theta \theta}}{\gamma_{r r}}, \\
\Gamma_{\phi \phi}^{r}=-\frac{1}{2} \frac{\sin ^{2} \theta \partial_{r} \gamma_{\theta \theta}}{\gamma_{r r}}, & \Gamma_{r \theta}^{\theta}=\frac{1}{2} \frac{\partial_{r} \gamma_{\theta \theta}}{\gamma_{\theta \theta}}, \\
\Gamma_{\phi \phi}^{\theta}=-\sin \theta \cos \theta, & \Gamma_{r \phi}^{\phi}=\frac{1}{2} \frac{\partial_{r} \gamma_{\theta \theta}}{\gamma_{\theta \theta}}, \\
\Gamma_{\theta \phi}^{\phi}=\cot \theta, &
\end{array}
$$

which will become useful also to calculate the Ricci tensor components of $\Sigma_{t}$.

We also need explicit expressions for the constraints. In the Hamiltonian constraint, aside of $K$ and $K_{i j} K^{i j}$, one needs the expression for $R$. The Ricci tensor is $R_{i j}=$ $\operatorname{diag}\left(R_{r r}, R_{\theta \theta}, \sin ^{2} \theta R_{\theta \theta}\right)$ whose components and trace are

$$
\begin{aligned}
R_{r r} & =-\frac{\partial_{r r} \gamma_{\theta \theta}}{\gamma_{\theta \theta}}+\frac{1}{2} \frac{\left(\partial_{r} \gamma_{\theta \theta}\right)^{2}}{\gamma_{\theta \theta}^{2}}+\frac{1}{2} \frac{\left(\partial_{r} \gamma_{r r}\right)\left(\partial_{r} \gamma_{\theta \theta}\right)}{\gamma_{r r} \gamma_{\theta \theta}}, \\
R_{\theta \theta} & =-\frac{1}{2} \frac{\partial_{r r} \gamma_{\theta \theta}}{\gamma_{r r}}+1+\frac{1}{4} \frac{\partial_{r} \gamma_{r r} \partial_{r} \gamma_{\theta \theta}}{\gamma_{r r}^{2}}, \\
R & =\gamma^{i j} R_{i j}=-2 \frac{\partial_{r r} \gamma_{\theta \theta}}{\gamma_{r r} \gamma_{\theta \theta}}+\frac{1}{2} \frac{\left(\partial_{r} \gamma_{\theta \theta}\right)^{2}}{\gamma_{r r} \gamma_{\theta \theta}^{2}} \\
& +\frac{\partial_{r} \gamma_{r r} \partial_{r} \gamma_{\theta \theta}}{\gamma_{r r}^{2} \gamma_{\theta \theta}}+\frac{2}{\gamma_{\theta \theta}} .
\end{aligned}
$$

The Momentum constraint additionally needs the terms $D_{j} K^{i j}$ and $D_{j}\left(\gamma^{i j} K\right)=\gamma^{i j} D_{j} K$, which for $i=r$ read

$$
\begin{aligned}
D_{j} K^{r j} & =\partial_{r}\left(\frac{K_{r r}}{\gamma_{r r}^{2}}\right)+2 \frac{K_{r r}}{\gamma_{r r}^{2}} \Gamma_{r r}^{r}+\frac{K_{r r}}{\gamma_{r r}^{2}}\left(\Gamma_{r \theta}^{\theta}+\Gamma_{r \phi}^{\phi}\right) \\
& +\frac{K_{\theta \theta}}{\gamma_{\theta \theta}^{2}} \Gamma^{r}{ }_{\theta \theta}+\frac{K_{\phi \phi}}{\gamma_{\phi \phi}^{2}} \Gamma_{\phi \phi}^{r}=\frac{\partial_{r} K_{r r}}{\gamma_{r r}^{2}} \\
& -\frac{K_{r r} \partial_{r} \gamma_{r r}}{\gamma_{r r}^{3}}+\frac{K_{r r} \partial_{r} \gamma_{\theta \theta}}{\gamma_{r r}^{2} \gamma_{\theta \theta}}-\frac{K_{\theta \theta} \partial_{r} \gamma_{\theta \theta}}{\gamma_{\theta \theta}^{2} \gamma_{r r}}, \\
& =\frac{\partial_{r} K_{r r}}{\gamma_{r r}^{2}}-\frac{K_{r r} \partial_{r} \gamma_{r r}}{\gamma_{r r}^{3}} \gamma^{r j} D_{j} K=\frac{\partial_{r} K}{\gamma_{r r}} \\
& +2 \frac{\partial_{r} K_{\theta \theta}}{\gamma_{r r} \gamma_{\theta \theta}}-2 \frac{K_{\theta \theta} \partial_{r} \gamma_{\theta \theta}}{\gamma_{r r} \gamma_{\theta \theta}^{2}} .
\end{aligned}
$$

Then the expressions for the two non-trivial constraints (35.36) are written as

$$
\begin{aligned}
\mathcal{H} & :=2\left(\frac{K_{\theta \theta}}{\gamma_{\theta \theta}}\right)^{2}+4 \frac{K_{r r} K_{\theta \theta}}{\gamma_{r r} \gamma_{\theta \theta}}+\frac{\gamma_{r r}^{\prime} \gamma_{\theta \theta}^{\prime}}{\gamma_{r r}^{2} \gamma_{\theta \theta}}+\frac{2}{\gamma_{\theta \theta}} \\
& +\frac{\gamma_{\theta \theta}^{\prime 2}}{2 \gamma_{r r} \gamma_{\theta \theta}^{2}}-2 \frac{\gamma_{\theta \theta}^{\prime \prime}}{\gamma_{r r} \gamma_{\theta \theta}}-16 \pi \rho=0, \\
\mathcal{M}^{r} & :=-2 \frac{K_{\theta \theta}^{\prime}}{\gamma_{r r} \gamma_{\theta \theta}}+\frac{K_{\theta \theta} \gamma_{\theta \theta}^{\prime}}{\gamma_{r r} \gamma_{\theta \theta}^{2}} \\
& +\frac{K_{r r} \gamma_{\theta \theta}^{\prime}}{\gamma_{r r}^{2} \gamma_{\theta \theta}}-8 \pi S^{r}=0 .
\end{aligned}
$$


These expressions will be important in two ways: i) they will be used for the construction of consistent initial conditions for the IVP and ii) used to monitor whether or not the numerical solution is consistent in the continuum limit with Einstein's equations.

\subsection{The evolution problem}

It is time to wrap up the ingredients constructed so far. The goal is to solve the evolution problem for matter variables and geometry simultaneously. Evolution equations are the equations for a perfect flui (15), this time with geometric factors evolving in time according to the ADM Eqs. (41 44).

Notice that geometric factors affect the flu es in (15) for the evolution of the flui through $\alpha, \beta$ and $\sqrt{\gamma}$, and also the sources (14) that depend on the time-changing Christoffel symbols of the space-time metric (10). These symbols appear in Appendix 5., and are different from those exclusive for the EF metric (29), which is stationary, and from those for solely the hypersurfaces (46).

Conversely, the time-dependent matter sources affect the evolution of metric and curvature components in (41. 44) through $\rho, S_{r r}, S_{\theta \theta}, S$. We say that matter and space-time evolution is fully coupled.

For the solution to be consistent with Einstein's equations, it is necessary to set initial conditions that are solution of Einstein's equations. In order to guarantee this condition, one needs to set an initial matter distribution consistent with the space-time geometry that satisfie the constraint Eqs. $(48,49)$ at initial time. Those initial data are used to evolve the system using Eqs. (15/4144).

\subsubsection{Initial conditions}

We have to solve the two equations $\mathcal{H}$ and $\mathcal{M}^{r}$ given a distribution of matter characterized by $\rho_{0}, v^{r}, p, e$ at initial time, for the four variables $\gamma_{r r}, \gamma_{\theta \theta}, K_{r r}, K_{\theta \theta}$. This system is underdetermined, with two equations and four unknowns, and there are various strategies to solve it (see, e.g, [22,23]). Here we solve this problem by assuming an ansatz for two variables and solving for the two remaining ones. We choose to set

$$
\begin{aligned}
\gamma_{\theta \theta} & =r^{2} \\
K_{r r} & =-\frac{2 M}{r^{2}} \frac{1+\frac{M}{r}}{\sqrt{1+\frac{2 M}{r}}},
\end{aligned}
$$

which are the expressions for the Eddington-Finkelstein metric detailed in Appendix A. Then constraints $(48,49)$ become the following two equations for $\gamma_{r r}$ and $K_{\theta \theta}$, assuming these variables depend on $r$ only, at initial time

$$
\begin{aligned}
\gamma_{r r}^{\prime} & =\left(1-\gamma_{r r}\right) \frac{\gamma_{r r}}{r}-\frac{K_{\theta \theta}^{2}}{r^{3}} \gamma_{r r}^{2} \\
& -2 \gamma_{r r} \frac{K_{r r} K_{\theta \theta}}{r}+8 \pi r \gamma_{r r}^{2} \rho, \\
K_{\theta \theta}^{\prime} & =\frac{r}{\gamma_{r r}} K_{r r}+\frac{K_{\theta \theta}}{r}-4 \pi r^{2} \gamma_{r r} S^{r} .
\end{aligned}
$$

These can be solved using an ODE integrator across the spatial domain $r \in\left[r_{\min }, r_{\max }\right]$. In our case we use a fourth order Runge-Kutta method. As initial conditions for these two equations, we use the values for $\gamma_{r r}$ and $K_{\theta \theta}$ in (28) and the Appendix A, evaluated at $r_{\min }$

$$
\begin{aligned}
\gamma_{r r}\left(0, r_{\min }\right) & =1+\frac{2 M}{r_{\min }}, \\
K_{\theta \theta}\left(0, r_{\min }\right) & =\frac{2 M}{\sqrt{1+\frac{2 M}{r_{\min }}}},
\end{aligned}
$$

corresponding to the EF space-time.

For this, the flui properties need to be prescribed. As an example, we consider the matter and velocity distributions to be

$$
\begin{aligned}
\rho_{0} & =\max \left(A e^{-\left(r-r_{0}\right)^{2} / \sigma^{2}}, \rho_{a t m}\right), \\
v^{r} & = \begin{cases}v_{0} & \text { if } \rho_{0}>\rho_{a t m} \\
0 & \text { otherwise }\end{cases} \\
e & =e_{0},
\end{aligned}
$$

and from here one can construct $p=\rho_{0} e(\Gamma-1), h=$ $1+e+p / \rho_{0}$, whereas $W=1 / \sqrt{1-\gamma_{r r} v^{r} v^{r}}$, needed to calculate the matter quantities $\rho$ and $S^{r}$ in (40), contains the unknown $\gamma_{r r}$, and then incorporated into (51). The reason to choose a region with non-zero initial velocity is that, as described in section 2.1. these methods need a non-zero density that works as a background atmosphere, and we simply do not want the atmosphere to move at the beginning.

\subsubsection{Numerical methods for the evolution of geometry}

The problem consists in solving the Euler equations in (15) at the same time as the evolution equations of geometry (41 44) on the same discrete domain. We use the same domain used for the test fiel case that we rewrite here, with $r_{\min }=1$, $r_{\max }=51$, and defin the discrete domain as the set of points $\left(t^{n}, r_{i}\right)$ such that $r_{i}=r_{\min }+i \Delta r, i=0,1,2, \ldots, N_{r}$ and $t^{n}=n \Delta t, n=0,1,2, \ldots, N_{t}$ such that $t_{f}=N_{t} \Delta t$. The spatial resolution is $\Delta r=\left(r_{\max }-r_{\min }\right) / N_{r}$, and time resolution $\Delta t=0.25 \Delta r$. Notice that for the geometry the numerical domain is vertex-centered, and at the same time we use this numerical domain for the finit volume discretization used for hydrodynamics equations. One only has to be careful with keeping in mind that vertices at $\left(t^{n}, r_{i}\right)$ correspond 
to a corner shared by the $L$ cell, centered at $\left(t^{n+1 / 2}, r_{i-1 / 2}\right)$ and the $R$ cell centered at $\left(t^{n+1 / 2}, r_{i+1 / 2}\right)$.

We solve Eqs. (41 44) for the evolution of geometry using the method of lines (MoL), with second order accurate expressions for the space derivatives on the right hand sides and a third order integrator in time. Moreover we discretize advection terms with speed $\beta^{r}$ with causally connected stencils. The details are described in Appendix B.

\subsubsection{Gauge}

The evolution of gauge functions $\alpha$ and $\beta^{r}$ can be driven by evolution equations that prevent the grid stretching that promotes the formation of gauge shocks or singularities of geometric quantities [22,23]. Nevertheless, here we use a more basic and illustrative gauge control, requiring $\alpha$ and $\beta^{r}$ to obey some simple conditions.

Condition 1. The space-time is being foliated with hypersurfaces $\Sigma_{t}$, which in turn are foliated with spheres of area $4 \pi \gamma_{\theta \theta}$ and radial lines of constant $(\theta, \phi)$. For simplicity we request the condition that the area of spheres to be $4 \pi r^{2}$, that is, we set $\gamma_{\theta \theta}=r^{2}$ as we did for the initial conditions, but we want to keep it that way during the evolution. Then we force the right hand side of Eq. (42) to be zero, that is $\alpha$ is forced to obey the condition

$$
-2 \alpha K_{\theta \theta}+\beta^{r} \partial_{r} \gamma_{\theta \theta}=0 .
$$

Condition 2. We want to keep the ingoing radial null rays to have slope -1 like in Fig. 1, a convenient property of EF coordinates is that it helps with keeping the light cones open near the event horizon of the black hole, even if the spacetime is not exactly that of EF. This condition can be obtained from the equation for radial null rays in metric (10)

$$
\begin{aligned}
d s^{2} & =\left(-\alpha^{2}+\gamma_{r r} \beta^{r} \beta^{r}\right) d t^{2}+2 \gamma_{r r} \beta^{r} d r d t+\gamma_{r r} d r^{2}=0 \\
& \Rightarrow\left(-\alpha^{2}+\gamma_{r r} \beta^{r} \beta^{r}\right)\left(\frac{d t}{d r}\right)^{2}+2 \gamma_{r r} \beta^{r} \frac{d t}{d r}+\gamma_{r r}=0
\end{aligned}
$$

assuming the condition $d t / d r=-1$ one has

$$
\alpha= \pm \sqrt{\gamma_{r r}}\left(1-\beta^{r}\right),
$$

with the positive sign corresponding to ingoing rays. Solving (54) and (55) for $\alpha$ and $\beta^{r}$ one obtains

$$
\begin{aligned}
\beta^{r} & =\frac{2 \sqrt{\gamma_{r r}} K_{\theta \theta}}{2 \sqrt{\gamma_{r r}} K_{\theta \theta}+\gamma_{\theta \theta}^{\prime}}, \\
\alpha & =\sqrt{\gamma_{r r}}\left(1-\beta^{r}\right) .
\end{aligned}
$$

We enforce these conditions to hold during the evolution after every step during time-integration with the MoL.

\subsubsection{Boundary conditions}

Inner boundary conditions. Due to the causal structure inside the horizon, where the light cones point inwards, similar to those in Fig. 1, we use the same condition as for the flui in the fi ed background accretion case, that is, we extrapolate the values of both, conservative variables $D, J_{r}, \tau$ for the matter and $\gamma_{r r}, \gamma_{\theta \theta}, K_{r r}, K_{\theta \theta}$ for the geometry at $r_{m i n}$, as done in (30).

Outer boundary conditions. We start with the conditions for the geometry. It is common to consider that metric and curvature components behave like waves moving at the speed of light, far enough from the zones of strong gravitational field in our case far from the black hole horizon. In particular, it is assumed that if the system is isolated the waves travel outwards. Here we follow this assumption for geometric functions. Any of these quantities is represented by $\psi=\psi(t, r)$, then it obeys the wave equation $\square \psi=0$ with $\square$ the D'Alambert operator for Minkowski space-time, which is appropriate for a far enough external boundary. In spherical coordinates the spherical wave operator in Minkowski spacetime can be factorized as follows

$$
\begin{aligned}
\square \psi & =\frac{\partial^{2} \psi}{\partial t^{2}}-c^{2} \nabla^{2} \psi=\frac{\partial^{2} \psi}{\partial t^{2}}-c^{2}\left(\frac{\partial^{2} \psi}{\partial r^{2}}+\frac{2}{r} \frac{\partial \psi}{\partial r}\right) \\
& =\left[\frac{\partial}{\partial t}+c_{-}\left(\frac{\partial}{\partial r}+\frac{1}{r}\right)\right]\left[\frac{\partial}{\partial t}-c_{-}\left(\frac{\partial}{\partial r}+\frac{1}{r}\right)\right] \psi=0,
\end{aligned}
$$

where the firs factor corresponds to the mode moving inward and the second one corresponds to the outgoing mode. Since we want the outgoing mode to survive, we must set the ingoing mode to zero, that is, we impose the condition

$$
\left[\frac{\partial}{\partial t}+c_{-}\left(\frac{\partial}{\partial r}+\frac{1}{r}\right)\right] \psi=0,
$$

for $\psi$. Now, the velocity $c_{-}$is the velocity of light written in terms of the space-time coordinates at $r=r_{\max }$. This velocity results from the null ray condition $d s^{2}=\left(-\alpha^{2}+\right.$ $\left.\gamma_{r r} \beta^{r} \beta^{r}\right) d t^{2}+2 \gamma_{r r} \beta^{r} d r d t+\gamma_{r r} d r^{2}=0$ as above, but now solving for

$$
c_{ \pm}=\left.\left(\frac{d r}{d t}\right)\right|_{r_{\max }}=\left.\left(-\beta^{r} \mp \frac{\alpha}{\sqrt{\gamma_{r r}}}\right)\right|_{r_{\max }} .
$$

Now the question is who is $\psi$. One possibility is to apply this condition to the metric and curvature components $\psi=\gamma_{r r}, \gamma_{\theta \theta}, K_{r r}, K_{\theta \theta}$. However, following [15], we defin the evolution of $\gamma_{r r}, \gamma_{\theta \theta}, K_{r r}, K_{\theta \theta}$ assuming the departure of these functions from a certain background space-time is a wave, that is, we impose the evolution of geometrical variables as follows

$$
\frac{\partial \psi}{\partial t}=-c_{-}\left(\frac{\partial}{\partial r}+\frac{1}{r}\right) \tilde{\psi}
$$

where $\psi$ can be $\gamma_{r r}, \gamma_{\theta \theta}, K_{r r}, K_{\theta \theta}$, and $\tilde{\psi}=\psi-\psi_{b g}$. Since the space-time we deal with is similar to that of EF, we choose the background metric and curvature components to be those of EF, whose formulas are in (28) and in Appendix D.

Now, the boundary condition for matter at $r_{\max }$ is out$\mathrm{fl} \mathrm{w}$, so that the flui can get off the domain. This is implemented by copying the values of the conservative variables $D, J_{r}, \tau$ at point $N_{r}$ from their values at point $N_{r}-1$ of $\mathcal{D}_{d}$. 


\subsection{Example}

We implement the methods described above using various resolutions with $N_{r}=2000,4000,8000$ or equivalently $\Delta r=0.025,0.0125,0.00625$, whereas time is discretized with resolution $\Delta t=0.25 \Delta r$. We set $\rho_{a t m}=10^{-13}$.

For initial data in (53) we use a pulse with $A=10^{-4}$, $r_{0}=20, \sigma=0.5, v_{0}=-0.1, e_{0}=0.01$ and consider a relativistic gas with $\Gamma=4 / 3$. The results of the solution are summarized in Fig. 5, where we show the dynamics of $\rho_{0}, p$, $v^{r}, \gamma_{r r}, K_{r r}$ and $K_{\theta \theta}$.

Let us firs describe the initial conditions. Notice that the rest mass density and pressure show the Gaussian profile whereas the velocity fiel has a square pulse shape. The metric function $\gamma_{r r}$ and $K_{\theta \theta}$ bent near $r=20$ at the center of the Gaussian for matter, whereas $\gamma_{\theta \theta}$ and $K_{r r}$ are those of the EF metric as prescribed before. Notice that, according to (53) the velocity is discontinuous at two points, where the Gaussian density coincides with the atmosphere value.

One of those points, the one at the right, evolves as a shock, discontinuous in density, pressure and velocity as can be seen in the snapshots. The one at the left evolves with a smooth density and pressure, but discontinuous in velocity. After initial time the pulse expands due to pressure and redistributes across the numerical domain, part of the gas toward the black hole, where $v^{r}<0$ and part trying to escape in regions where $v^{r}>0$. Eventually the density decreases in amplitude, which means that it has been absorbed by the black hole and finall escaped through the boundary at $r_{\min }$. Notice from the plot for $\rho_{0}$ that the amount of matter moving to the right is small compared to that being accreted by the black hole.

With time $\rho_{0}, p$ and $v^{r}$ approach a stationary state with profile very similar to those for the accretion on the fi ed background in Fig. 3, and although small not completely the value of $\rho_{a t m}$. This is a parasitic accretion of the fluid which is unavoidable with the finit volume approach and is due to the presence of the atmosphere. This means that the atmosphere is all the time contributing with a small amount to the accretion process but is unphysical. In order to see how much this remanent is due to the atmosphere we solved the system for initial conditions with $\rho_{0}=\rho_{a t m}$ and zero velocity initially. This is pretty similar to the case we solved with the background fi ed, but this time the geometry evolves. In Fig. 6 we show the comparison between the remanent density profil after the Gaussian has been evolved in our example above and the density profil resulting from the evolution of only the atmosphere. The two profile are very similar, but not the same, and the profil of only the atmosphere has a smaller $\rho_{0}$ than that of the remanent. This indicates that part of the matter in the Gaussian pulse is never accreted nor escaped from the black hole and can be due to the accumulation of numerical errors.

Later on we will measure the amount of matter accreted during the process and see whether or not the Gaussian was accreted by the black hole.

As for the geometry in Fig. 5, the metric functions and extrinsic curvature components expel the bump set at initial time due to the Gaussian shell of matter.

As far as the numerical solution is concerned, up to here the problem can be said it has been solved, nevertheless, the physics and numerical reliability of the solution require some extra work we describe now.

\subsection{Diagnostics}

\subsubsection{Convergence of constraints}

Notice that we are not solving the whole set of Einstein's equations, only those for the evolution of the metric and extrinsic curvature components, whereas the constraints have been solved only at initial time. This is not a particular issue of the approach here, but it is a common signature of General Relativistic simulations based on the $3+1$ decomposition of the space-time: there are evolution equations solved as an Initial Value Problem, and constraint equations used to monitor the reliability of the solutions [22,23], in fact, formulations other than ADM, defin constraints additional to the Hamiltonian and Momentum Constraints, which may also be monitored.

Constraint Eqs. (48) and (49) have to be fulfille in the continuum in order for the numerical solution of evolution equations to be consistent with Einstein's equations. For this we have to check that in the continuum limit $\mathcal{H}$ and $\mathcal{M}^{r}$ converge to zero. For this we apply a convergence test as follows. Suppose we calculate $\mathcal{H}_{1}$ when we solve the equations using a base resolution $\Delta r_{1}=\Delta r$, then we construct a second solution $\mathcal{H}_{2}$ using resolution $\Delta r_{2}=\Delta r_{1} / 2$. Assuming the dominating global error of the solution, with all the methods used for hydrodynamics and for geometry are of order $n$, and noticing that the errors depending on time resolution $\Delta t=C \Delta r$ are already encrypted in the errors in $\Delta r$, we can write that

$$
\begin{aligned}
\mathcal{H}_{1} & =\mathcal{H}_{0}+E \Delta r^{n}, \\
\mathcal{H}_{2} & =\mathcal{H}_{0}+E\left(\frac{\Delta r}{2}\right)^{n},
\end{aligned}
$$

where $E$ is the amplitude of the error that depends on both, space and time, and $\mathcal{H}_{0}$ is the value of the constraint in the continuum limit. Dividing these two expressions and substituting that $\mathcal{H}_{0}=0$, that is, the constraint is fulfille in the continuum, one find that

$$
\frac{\mathcal{H}_{1}}{\mathcal{H}_{2}} \simeq 2^{n},
$$

is the Convergence Factor (CF) of the numerical solutions. The time integrator of the MoL is third order accurate, finit differences for the right hand sides of Eqs. (41 44) is second order accurate, and the HRSC methods of hydrodynamics are second order accurate for smooth fl ws and firs order near discontinuities, in particular the outgoing shock wave. Thus, depending on the part of the domain, $n$ should be in theory 
a)
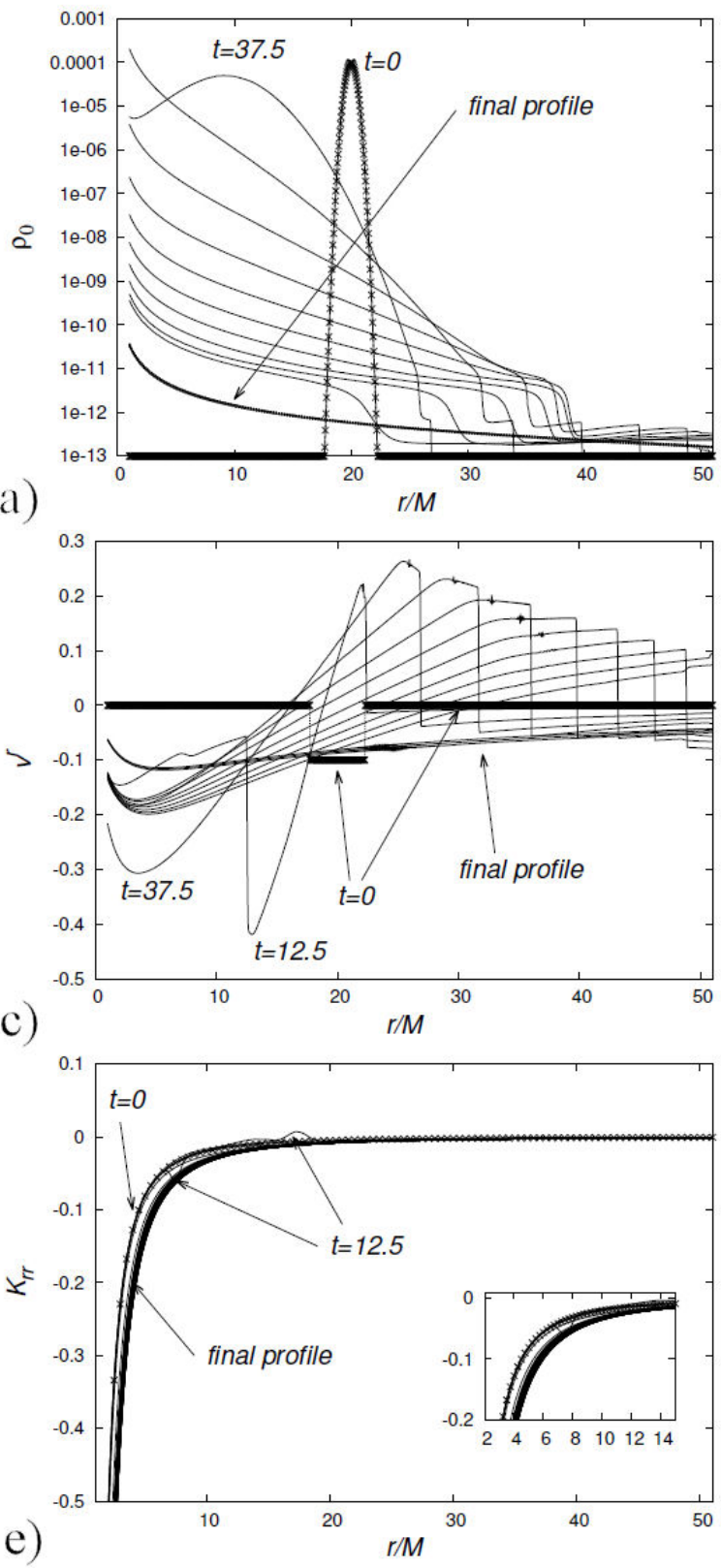
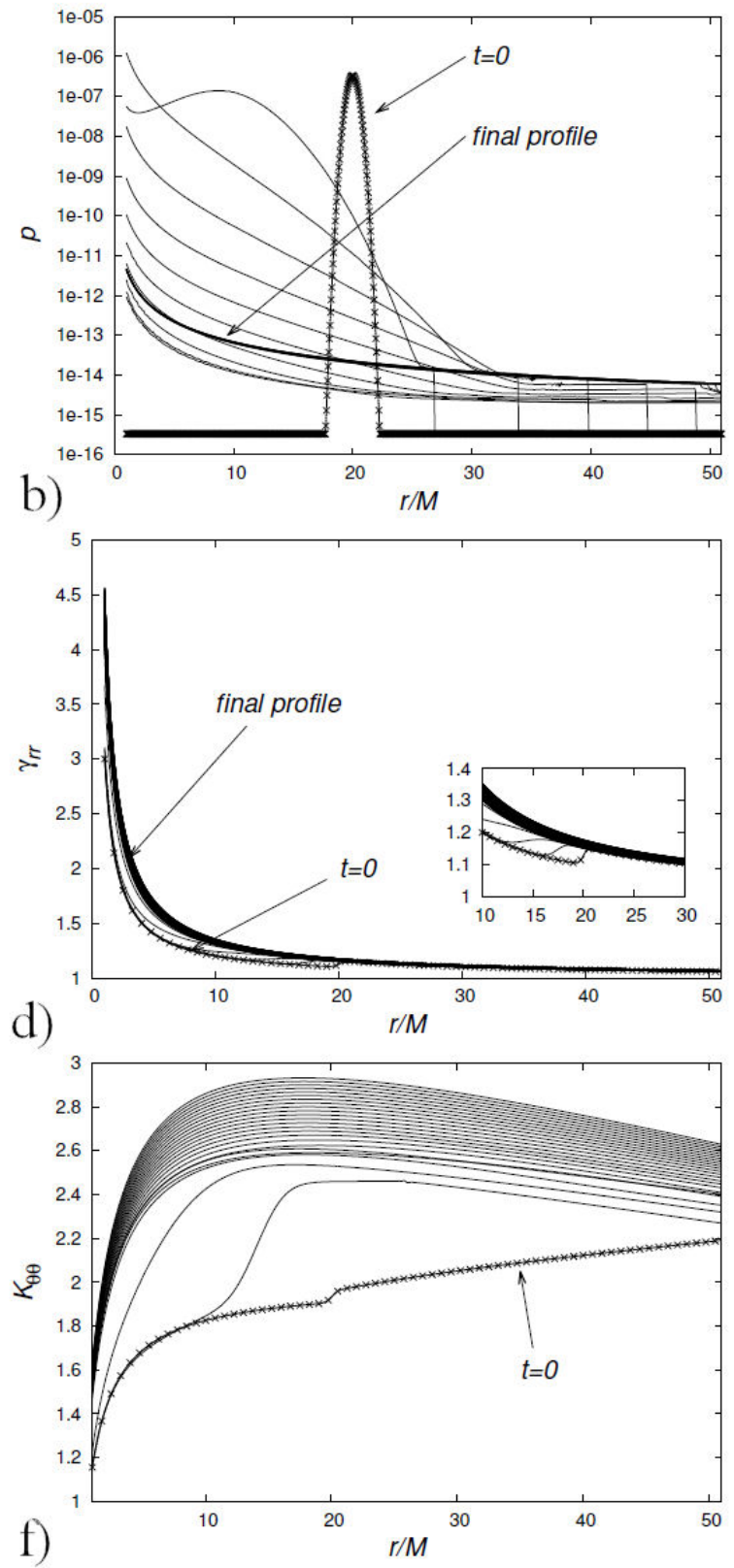

FIGURE 5. Snapshots of $\rho_{0}, p, v^{r}, \gamma_{r r}, K_{r r}$ and $K_{\theta \theta}$ for the accretion of a Gaussian shell. Notice that matter and geometry evolve in time and become nearly stationary after the pulse has been absorbed by the inner boundary within the black hole's horizon. These results correspond to the solution with $\Delta r=0.0125$. The lines with points indicate the initial values for each variable. As an example with units, this solution for a black hole of initial horizon mass $M=100 M_{\odot}$ the range of $\rho_{0}$ in cgs units is from 6.177 to $6.177 \times 10^{10} \mathrm{gr} / \mathrm{cm}^{3}$, the velocity in units of $c$ and the range of pressure axis is from $5.55 \times 10^{18}$ to $5.55 \times 10^{29} \mathrm{gr} / \mathrm{cm} / \mathrm{s}^{2}$.

$1 \leq n \leq 2$. That is, the violation of the Hamiltonian constraint, that now we see depends on resolution, should approximately fulfil relation $(60)$. We say the relation $(60)$ has to be fulfille approximately because in (59) the expansion is truncated.

Now, checking whether the constraint converges at every time is rather unpractical, one would need to check this relation at every point of the space-time domain. Based on the Lax Theorem [28], we in principle can use the convergence of a norm, for example $L_{\infty}, L_{1}$ or $L_{2}$. We choose the $L_{2}$ norm of $\mathcal{H}$ is calculated as

$$
L_{2}(\mathcal{H})=\sqrt{\int_{r_{\min }}^{r_{\max }}|\mathcal{H}|^{2} d r},
$$

integrated on the numerical domain along the radial direction. Then $L_{2}(\mathcal{H})$ can be calculated at each time and then used to track the convergence of the numerical solution. 


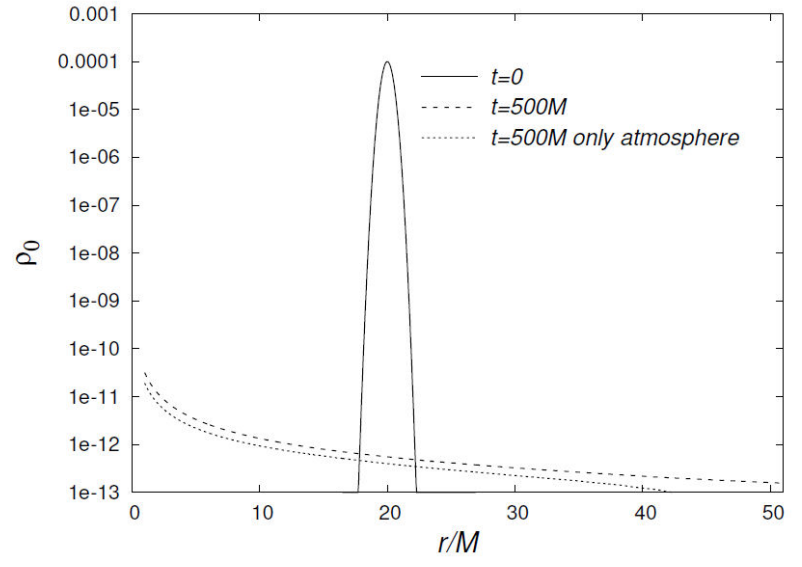

FIGURE 6 . We show the remanent of the rest mass density $\rho_{0}$ and compare its profil with the evolution of purely the atmosphere.

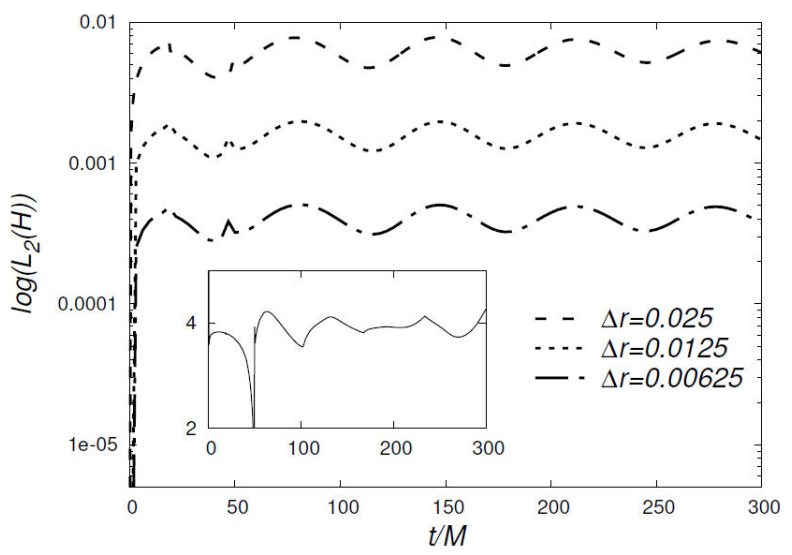

FIgURE 7. Convergence of $L_{2}(\mathcal{H})$ to zero as a function of time in the continuum limit. The factor between curves varies between 2 and 4 . In the inset we show the convergence factor of the $L_{2}$ norm of $\mathcal{H}$ using $\Delta r=0.025$ and 0.0125 , which implies that $(60)$ is fulfille between $n=1$ and $n=2$.

We show in Fig. 7 the convergence test of $L_{2}(\mathcal{H})$ for three different resolutions. We use a log scale in order to include more than two resolutions. The CF of $L_{2}(\mathcal{H})$ between two consecutive resolutions is close to 4 , which confirm that the dominant accuracy of the numerical solution is of order $n \simeq 2$.

\subsubsection{Apparent horizon}

An Apparent Horizon (AH) is a marginally trapped 2dimensional surface embedded within the hypersurface $\Sigma_{t}$, on which the expansion $\Theta$ of outgoing null geodesics is zero [23]. For the spherically symmetric space-time with metric (10) the expansion reads

$$
\Theta=\frac{\partial_{r} \gamma_{\theta \theta}}{\sqrt{\gamma_{r r}} \gamma_{\theta \theta}}-2 \frac{K_{\theta \theta}}{\gamma_{\theta \theta}} .
$$

In this case the $\mathrm{AH}$ is a two-sphere of radius $r_{A H}$. The marginally nature means that $r_{A H}$ is the outermost value of
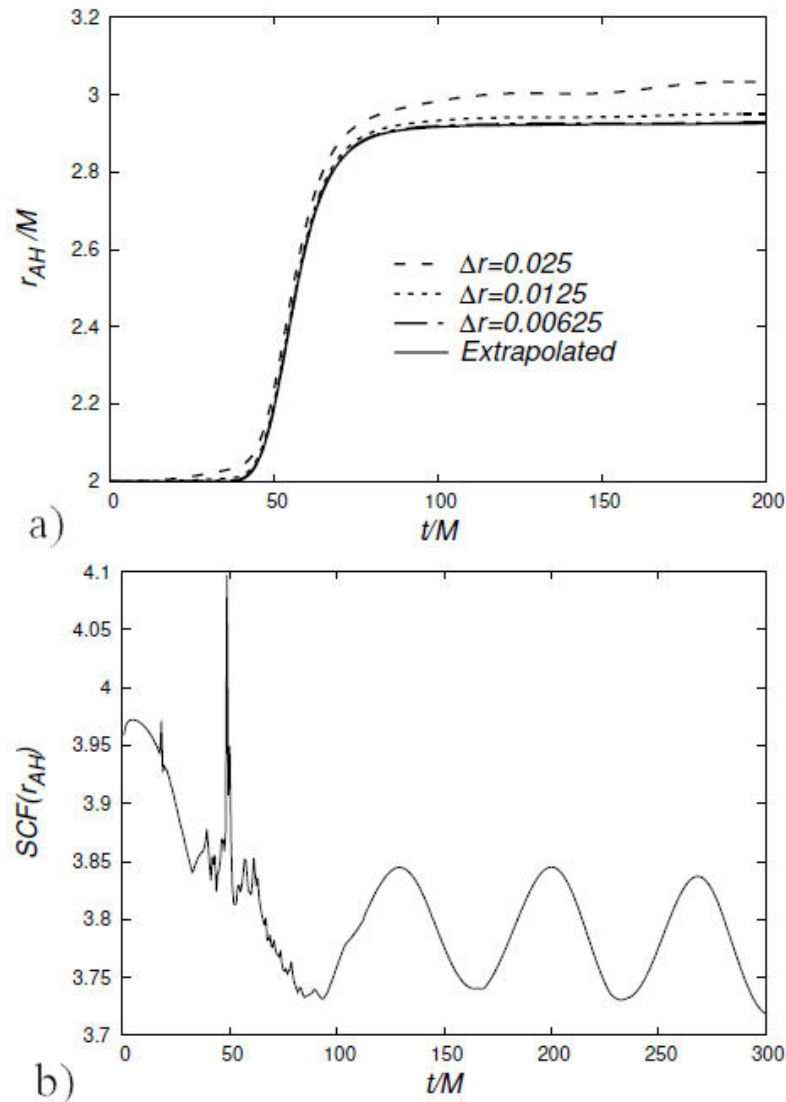

FIGURE 8. In the top we show the Apparent Horizon radius $r_{A H}$ as a function of time using various resolutions. We also show the Richarson extrapolation of the radius, which is constructed with the two fines resolutions and formula (64). In the bottom we show the self-convergence factor of $r_{A H}$ calculated using the three resolutions and formula (63). The spike appears at the moment when the horizon starts growing.

$r$ at which $\Theta=0$, for which we search for zeroes of $\Theta$ from $r_{\text {max }}$ inwards.

Formally the areal radius of the apparent horizon is $R_{A H}=\sqrt{\gamma_{\theta \theta}\left(t, r_{A H}\right)}$, located where the outermost zero of $\Theta$ is found. Then we calculate the area of the $\mathrm{AH}$ as that of the corresponding 2-sphere $A_{A H}=4 \pi R_{A H}^{2}$ and the $\mathrm{AH}$ mass $M_{A H}=R_{A H} / 2$ [22,23]. In our case, we are enforcing the condition $\gamma_{\theta \theta}=r^{2}$, and therefore $r_{A H}=R_{A H}$.

The apparent horizon radius from our simulations with different resolutions is shown in Fig. 8. This is a real numerical simulation, and the plot must show how the apparent horizon can depend importantly on the resolution of the numerical domain. However one has to check whether the apparent horizon radius is numerically valid.

Unlike the convergence test for the Hamiltonian constraint above, in this case what we need is a self-convergence test for $r_{A H}$. Assume the unknown value in the continuum at a given $t$ is $r_{A H, 0}$, and that $r_{A H, 1}, r_{A H, 2}$ and $r_{A H, 3}$ are the apparent horizon radii calculated for numerical solutions using respectively $\Delta r_{1}=0.025, \Delta r_{2}=\Delta r_{1} / 2$ and $\Delta r_{3}=\Delta r_{2} / 2$. Then one can construct expressions for the 
numerical values in terms of the hypothetical value $r_{A H, 0}$ as follows:

$$
\begin{aligned}
& r_{A H, 1}=r_{A H, 0}+E \Delta r_{1}^{n}, \\
& r_{A H, 2}=r_{A H, 0}+E\left(\frac{\Delta r_{1}}{2}\right)^{n}, \\
& r_{A H, 3}=r_{A H, 0}+E\left(\frac{\Delta r_{1}}{4}\right)^{n},
\end{aligned}
$$

with $E$ the amplitude of the error. A combination of these expressions leads to the self-convergence factor (SCF)

$$
S C F=\frac{r_{A H, 1}-r_{A H, 2}}{r_{A H, 2}-r_{A H, 3}} \simeq 2^{n},
$$

where $n$ is the order of self-convergence. In Fig. 8 we also show the SCF for $r_{A H}$ that oscillates between 3.7 and 4, as expected, since the accuracy of our methods lies between $n=1$ and $n=2$. In fact we can extract $n=S C F / \log (2)$ that oscillates in time because SCF does.

Now that we know $r_{A H}$ self-converges with order $n$ that changes in time within the expected range $1 \leq n \leq 2$, we are authorized to apply a Richardson extrapolation for $r_{A H}$. The extrapolated value is nearly the horizon radius in the continuum limit. Two resolutions suffic to calculate an extrapolation, for instance $\Delta r_{2}$ and $\Delta r_{3}$. From the two last expressions in (62) one can obtain that

$$
r_{A H, 0} \simeq \frac{2^{n} r_{A H, 3}-r_{A H, 2}}{2^{n}-1},
$$

which is the extrapolated AH radius. This is the expected radius in the continuum limit that we also show in Fig. 8 with a continuous line.

\subsubsection{Event Horizon}

The Event Horizon on the other hand, is a 3-surface, which is the boundary between the outgoing null rays that can escape to future null infinit $\mathscr{I}^{+}$, and those that end up in the space-time singularity of the black hole. For a formal definition see $[3,22,23]$. In order to exactly locate the EH of a space-time, one needs to know the whole space-time. An example is Schwarzschild solution in Schwarzschild coordinates, which is static, and the event horizon is the 3 -surface consisting on the 2 -sphere of radius $2 M$ cross the domain of time $S^{2}(2 M) \times \mathbb{R}$.

In our problem of the evolution of a black hole, as well as in professional simulations of black holes mergers and other compact objects $[25,26]$, only a small portion of the spacetime is known. For example in the firs simulations of headon mergers of black holes, the event horizon looks like a pairof pants that lasts only a finit time, as can be seen in [29] and the cover of that number of Science [30].

Here we implement a practical approach to locating the $\mathrm{EH}$ of our black hole. For this we launch outgoing null rays generated from initial locations $r=g_{k}$ of the spatial domain

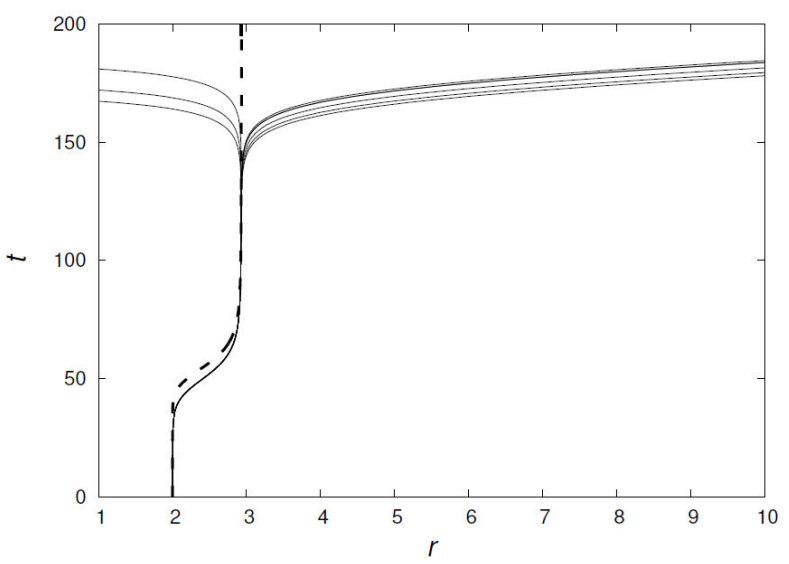

FIGURE 9. Bundle of outgoing null rays departing from points $r_{k}(0)=g_{k}$ near $r=2 M$ at initial time. The ray from which outgoing null rays depart outwards exponentially is the Event Horizon. The dashed line indicates the extrapolated Apparent Horizon radius for comparison. Notice that the $\mathrm{AH}$ lies inside the $\mathrm{EH}$ all the way.

and some of them will be able to escape to infinit, whereas others will end up pointing toward the black hole singularity. The limiting ray between these two behaviors will indicate within numerical accuracy, the location of the Event Horizon.

The evolution equation of a null ray is obtained from the condition $d s^{2}=\left(-\alpha^{2}+\gamma_{r r} \beta^{r} \beta^{r}\right) d t^{2}+2 \gamma_{r r} \beta^{r} d r d t+$ $\gamma_{r r} d r^{2}=0$ for radial rays. This time we search for an expression of $d r / d t$ :

$$
\begin{aligned}
d s^{2} & =\left(-\alpha^{2}+\gamma_{r r} \beta^{r} \beta^{r}\right) d t^{2}+2 \gamma_{r r} \beta^{r} d r d t+\gamma_{r r} d r^{2}=0 \\
& \Rightarrow \gamma_{r r}\left(\frac{d r}{d t}\right)^{2}+2 \gamma_{r r} \beta^{r} \frac{d r}{d t}+\left(-\alpha^{2}+\gamma_{r r} \beta^{r} \beta^{r}\right)=0,
\end{aligned}
$$

which implies

$$
\frac{d r}{d t}=-\beta^{r} \pm \frac{\alpha}{\sqrt{\gamma_{r r}}},
$$

for each null ray $k$ with points at $\left(t, r_{k}\right)$. The initial conditions for each geodesic $k$ are set to $r_{k}(0)=g_{k}$. In Fig. 9 we show a bundle of outgoing null rays departing from near the event horizon at initial time. For this particular bundle of geodesics, it can be noticed how the geodesics depart from each other at around $t \sim 150$, some of them moving toward the singularity and some others escaping to infinit .

For this figur we calculate these rays with the highest resolution solution $\Delta r=0.00625$, and also show the apparent horizon radius $r_{A H}$. Notice that the EH appears outside the apparent horizon and in fact starts growing earlier. This is a sign of the locality of the $\mathrm{AH}$, estimated at every $\Sigma_{t}$, and the global nature of the EH. A fina comment on the difference between $\mathrm{AH}$ and $\mathrm{EH}$ is that the $\mathrm{EH}$ is gauge independent, whereas the $\mathrm{AH}$ depends importantly on the gauge used to embed the spatial hypersurfaces into the space-time.

On the method. The method used here is rather tedious because one has to fine-tun the initial position of null geodesics that will be found to be close enough to each other 


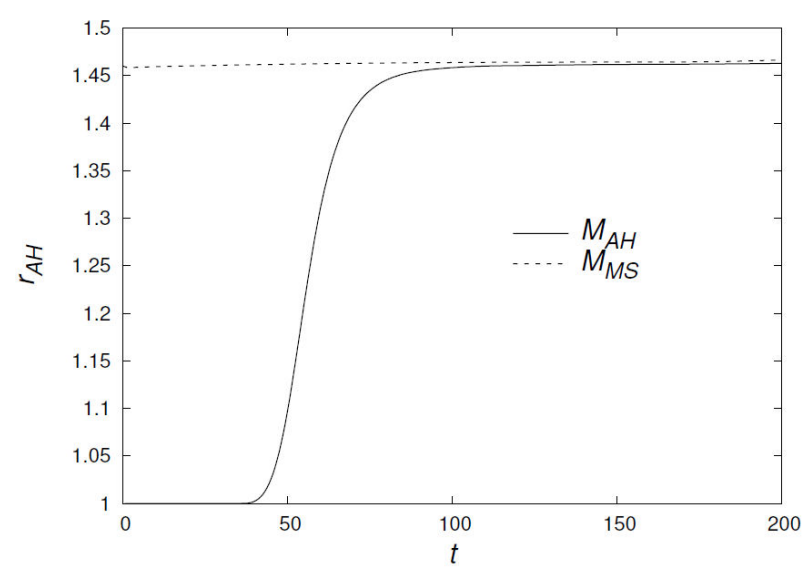

Figure 10. Time dependence of $M_{M S}$ and $M_{A H}$. Notice how the apparent horizon grows when the pulse is being accreted between $t \sim 40$ and $80 M$. Afterwards the apparent horizon mass approaches the Misner-Sharp mass, which indicates that black hole has accreted the flui in the domain.

and will depart only later in time. An advantage of our approach is that we solve the position equation of null rays (65) forward in time, simultaneously with matter and geometric variables using the MoL.

One more professional strategy consists in launching a bundle of null rays from the future starting at say $t=200$ and backwards in time, and the null ray to which they converge will mark the location of the event horizon. A considerable drawback is that one has to save in the hard disk the metric components at all times, which, depending on time resolution, can be a limitation, and the backward in time null-ray tracking has to be done at post-processing. This strategy is the one followed in the most general situations for the binary black hole and compact objects mergers, and actually captures the $\mathrm{EH}$ with any number of multipolar components, on which null surfaces launched backwards from the future converge to. This numerical tool, the Event Horizon Finder was developed within the code Cactus [31], and as far as we can tell, is a production tool used to track Event Horizons under fully non-linear 3D General Relativistic simulations [32]. This method has also been used in simpler applications, for example [33].

\subsubsection{Mass}

The Misner-Sharp mass is a useful quantity that serves to monitor the mass of a space-time slice $\Sigma_{t}$. For the $3+1$ metric used here, its expression reads [34]

$$
M_{M S}=\frac{1}{2} \sqrt{\gamma_{\theta \theta}}\left(1-\frac{1}{4} \frac{\left(\partial_{r} \gamma_{\theta \theta}\right)^{2}}{\gamma_{r r} \gamma_{\theta \theta}}+\frac{K_{\theta \theta}}{\gamma_{\theta \theta}}\right) \text {. }
$$

This mass accounts for the rest mass and energy contents of the slice and in the limit of $r \rightarrow \infty$ its value tends to the ADM mass of $\Sigma_{t}$. Here we use $M_{M S}$ to measure the energy content of the space-time in time during the evolution, and to see how the black hole horizon mass compares with it.
In Fig. 10 we show as functions of time, the apparent horizon mass $M_{A H}=r_{A H} / 2$ and $M_{M S}$. Notice that at initial time, the apparent horizon mass is one, whereas $M_{M S} \sim 1.458$. This means that the energy contents of the perfect flui is initially $\sim 0.458$. By time $t \sim 150 M$ the Gaussian shell is accreted and the horizon mass grows until it asymptotically approaches the value of $M_{M S}$. In practice the value of $M_{M S}$ is not constant, because there is the permanent atmosphere within the whole numerical domain that is being added up to the space-time mass. This is not important when the background space-time is fi ed, like in Michel accretion, but must be taken into account in full non-linear simulations.

\section{Final comments}

We have presented in detail the basic strategy and methods used to solve the time dependent accretion process of a perfect flui onto a black hole.

We expect this version of the problem to boost the starting point of students working on general relativity and relativistic astrophysics, to tackle more sophisticated accretion problems involving magnetohydrodynamics and radiation, ingredients that model the matter around black holes at current state of the art.

\section{Appendix}

\section{A. Christoffel symbols of the general spherically symmetric space-time}

Since the geometry is changing in time we need the symbols for the general space-time (10), not only for the EF spacetime (27) as we did in the test flui case. The non-zero Christoffel symbols for metric (10) are the following:

$$
\begin{aligned}
\Gamma_{t t}^{t} & =\frac{\left(\dot{\gamma}_{\mathrm{rr}}-2 \gamma_{\mathrm{rr}} \beta^{\mathrm{r} \prime}-\beta^{r} \gamma_{\mathrm{rr}}^{\prime}\right)\left(\beta^{r}\right)^{2}+2 \alpha\left(\dot{\alpha}+\alpha^{\prime} \beta^{r}\right)}{2 \alpha^{2}} \\
\Gamma_{t r}^{t} & =\frac{2 \alpha \alpha^{\prime}+\beta^{r}\left(\dot{\gamma}_{\mathrm{rr}}-2 \gamma_{\mathrm{rr}} \beta^{\mathrm{r} \prime}-\beta^{r} \gamma_{\mathrm{rr}}^{\prime}\right)}{2 \alpha^{2}}, \\
\Gamma_{r r}^{t} & =\frac{\dot{\gamma}_{\mathrm{rr}}-2 \gamma_{\mathrm{rr}} \beta^{\mathrm{r} \prime}-\beta^{r} \gamma_{\mathrm{rr}}^{\prime}}{2 \alpha^{2}}, \\
\Gamma_{\theta \theta}^{t} & =\frac{\dot{\gamma}_{\theta \theta}-\beta^{r} \gamma_{\theta \theta}^{\prime}}{2 \alpha^{2}}, \\
\Gamma_{\phi \phi}^{t} & =\sin ^{2} \theta \Gamma_{\theta \theta}^{t}, \alpha^{2} \\
\Gamma_{t t}^{r} & =\frac{1}{2}\left[\frac{\beta^{r}\left(\beta^{r}\left(\dot{\gamma}_{\mathrm{rr}} \beta^{r}+2 \gamma_{\mathrm{rr}} \dot{\beta}^{r}+2 \dot{\gamma}_{r r} \beta^{r}\right)-2 \alpha \dot{\alpha}\right)}{\alpha^{2}}\right. \\
& +\left(\frac{1}{\left.\gamma_{\mathrm{rr}}-\frac{\left(\beta^{r}\right)^{2}}{\alpha^{2}}\right)\left(-\gamma_{\mathrm{rr}}^{\prime}\left(\beta^{r}\right)^{2}+2\left(\dot{\gamma}_{\mathrm{rr}}-\gamma_{\mathrm{rr}} \beta^{\mathrm{r} \prime}\right) \beta^{r}\right.}\right. \\
+ & \left.\left.2 \alpha \alpha^{\prime}+2 \gamma_{\mathrm{rr}} \dot{\beta}^{r}\right)\right],
\end{aligned}
$$


$\Gamma_{t r}^{r}=\frac{1}{2}\left(\frac{\left(-\dot{\gamma}_{\mathrm{rr}}+2 \gamma_{\mathrm{rr}} \beta^{\mathrm{r} \prime}+\beta^{r} \gamma_{\mathrm{rr}}^{\prime}\right)\left(\beta^{r}\right)^{2}}{\alpha^{2}}-\frac{2 \alpha^{\prime} \beta^{r}}{\alpha}+\frac{\dot{\gamma}_{\mathrm{rr}}}{\gamma_{\mathrm{rr}}}\right)$

$\Gamma_{r r}^{r}=\frac{1}{2}\left(\frac{\gamma_{\mathrm{rr}}^{\prime}}{\gamma_{\mathrm{rr}}}+\frac{\beta^{r}\left(-\dot{\gamma}_{\mathrm{rr}}+2 \gamma_{\mathrm{rr}} \beta^{\mathrm{r} \prime}+\beta^{r} \gamma_{\mathrm{rr}}^{\prime}\right)}{\alpha^{2}}\right)$,

$\Gamma_{\theta \theta}^{r}=\frac{1}{2}\left(\frac{\beta^{r}\left(\beta^{r} \gamma_{\theta \theta}^{\prime}-\dot{\gamma}_{\theta \theta}\right)}{\alpha^{2}}-\frac{\gamma_{\theta \theta}^{\prime}}{\gamma_{\mathrm{rr}}}\right)$,

$\Gamma_{\phi \phi}^{r}=\sin ^{2} \theta \Gamma_{\theta \theta}^{r}$,

$\Gamma_{t \theta}^{\theta}=\frac{\dot{\gamma}_{\theta \theta}}{2 \gamma_{\theta \theta}}$

$\Gamma_{r \theta}^{\theta}=\frac{\gamma_{\theta \theta}^{\prime}}{2 \gamma_{\theta \theta}}$

$\Gamma_{\phi \phi}^{\theta}=-\cos \theta \sin \theta$,

$\Gamma_{t \phi}^{\phi}=\frac{\dot{\gamma}_{\theta \theta}}{2 \gamma_{\theta \theta}}$

$\Gamma_{r \phi}^{\phi}=\frac{\gamma_{\theta \theta}^{\prime}}{2 \gamma_{\theta \theta}}$

$\Gamma_{\theta \phi}^{\phi}=\cot \theta$,

where we used a prime to denote derivative with respect to $r$ and dot for time derivative.

An important practical detail is that in these expressions there are time derivatives of various functions. It is convenient to set the values for $\dot{\gamma}_{r r}$ and $\dot{\gamma}_{\theta \theta}$ to the right hand sides of their respective evolution Eqs. (41) and (42). The term $\dot{\alpha}$ is set to zero since there is no evolution equation for $\alpha$.

\section{B. Method of lines}

Assume one has to solve an Initial Value Problem involving a firs order in time differential equation define on a chunk of space-time described by the variables $t$ and $x$, and ruled by the evolution equation

$$
\frac{\partial f}{\partial t}=r h s\left(h, \frac{\partial g}{\partial x}\right)
$$

where $f=f(t, x), g=g(t, x), h=h(t, x)$ are functions define on the domain $\mathcal{D}=x \in\left[x_{\min }, x_{\max }\right] \times t \in\left[0, t_{f}\right]$, with some initial $f(0, x)=f_{0}(x)$ and boundary conditions $f\left(t, x_{\min }\right), f\left(t, x_{\max }\right)$ on $f$. The right hand side of the equation is a generic function. This is a type of equation we deal with in this paper, (15) and (41,44). An important property is that the equations of hydrodynamics and geometry are firs order in time and contain firs order and at most second order derivatives in space. Here we use firs order derivatives in space for illustration.

A discrete version of the problem requires the definitio of a discrete version of the domain $\mathcal{D}_{d}=\left\{\left(t^{n}, x_{i}\right) \mid x_{i}=\right.$ $\left.x_{\min }+i \Delta x, t=n \Delta t\right\}, i=0, \ldots, N_{x}, n=0,1, \ldots$, where $\Delta x=\left(x_{\max }-x_{\min }\right) / N_{x}$ is the spatial resolution and
$\Delta t=C \Delta x$ the time resolution. The functions involved in the equation, $f, g, h$ and their derivatives are define only at elements of $\mathcal{D}_{d}$. At each point $\left(t^{n}, x_{i}\right) \in \mathcal{D}_{d}$ one define the semi-discrete version of the Eq. (B.1) as follows:

$$
\left.\frac{\partial f}{\partial t}\right|_{\left(t^{k}, x_{i}\right)}=\operatorname{rhs}\left(\left.h\right|_{\left(t^{n}, x_{i}\right)},\left.\frac{\partial g}{\partial x}\right|_{\left(t^{n}, x_{i}\right)}\right) .
$$

This is an ODE for $f$ at each $x_{i}$, to be integrated from $t^{n}$ to $t^{n+1}$. In fact it is an IVP in the time domain $t \in\left[0, t_{f}\right]$ for each value $x_{i}$. This approach is called the Method of Lines (MoL), because the IVP associated to a PDE will be solved by integrating the set of $N_{x}+1$ ODEs along lines of constant position $x_{i}$ for $i=0, \ldots, N_{x}$.

The integration from $t^{n}$ to $t^{n+1}$ can be carried out using an integrator of ODEs, in our case a third order Runge-Kutta method. Then, the implementation of the MoL centers in the ability to construct the discrete version of $r h s$. In our equations we have spatial derivatives of firs and second order.

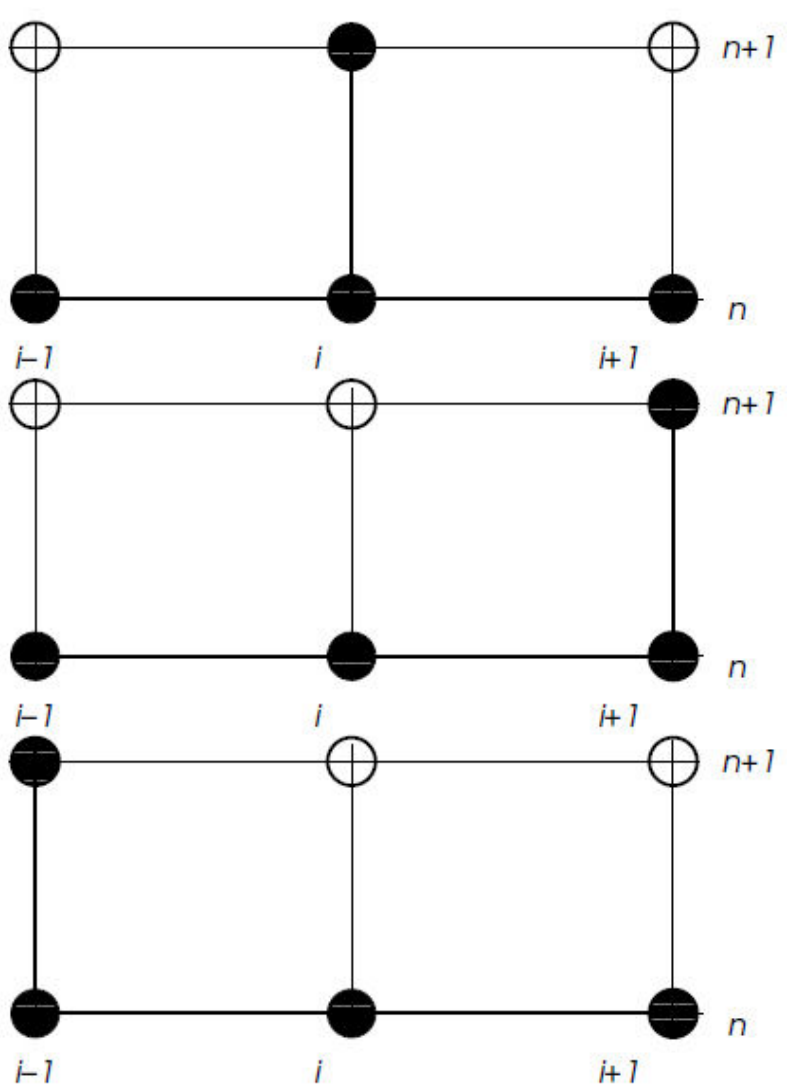

FIGURE B.1. We show the molecules used for the centered Finite Differences approximation with second order accuracy for $\beta^{r}=0$ at the top, the one-sided used for $\beta^{r}>0$ at the middle and the one-sided used for $\beta^{r}<0$ at the bottom. White circles indicate the points of $\mathcal{D}_{d}$ that are not used for the evolution step from $t^{n}$ to $t^{n+1}$ in each case. 
Expressions for the firs order derivative on $\mathcal{D}_{d}$ are

$$
\begin{aligned}
& \left.\frac{\partial g}{\partial x}\right|_{\left(t^{n}, x_{i}\right)}=\frac{g_{i+1}^{n}-g_{i-1}^{n}}{2 \Delta x}+\mathcal{O}\left(\Delta x^{2}\right), \\
& \left.\frac{\partial g}{\partial x}\right|_{\left(t^{n}, x_{i}\right)}=\frac{-3 g_{i}^{n}+4 g_{i+1}^{n}-g_{i+2}^{n}}{2 \Delta x}+\mathcal{O}\left(\Delta x^{2}\right), \\
& \left.\frac{\partial g}{\partial x}\right|_{\left(t^{n}, x_{i}\right)}=\frac{3 g_{i}^{n}-4 g_{i-1}^{n}+g_{i-2}^{n}}{2 \Delta x}+\mathcal{O}\left(\Delta x^{2}\right),
\end{aligned}
$$

obtained from Taylor expansions of $g$ around $x_{i}$ and along the spatial direction of the domain. Expression (B.3) is centered, and uses the information of $g$ at the two nearest neighbors of $\left(t^{n}, x_{i}\right)$. The later two expressions are one-sided because they use values of $g$ to the right or left from $\left(t^{n}, x_{i}\right)$.

For example, assume $r h s(h, \partial g / \partial x)=a \partial g / \partial x$, then the semi-discrete version of the equation would be

$$
\left.\frac{\partial f}{\partial t}\right|_{\left(x_{i}, t^{n}\right)}=a \frac{g_{i+1}^{n}-g_{i-1}^{n}}{2 \Delta x}+\mathcal{O}\left(\Delta x^{2}\right) .
$$

This version is commonly represented as a molecule, indicating the points of the domain involved to achieve a jump of $f$ from $t^{n}$ to $t^{n+1}$. In the particular case of (B.6) the molecule appears at the top in Fig. B.1.

MoL for HRSC methods. The centered expression is used for the rhs of $D, J_{r}, \tau(15)$ as follows. For example, the semi-discrete version of the evolution equation for the cell-centered value of $D$ is, from (20)

$$
\partial_{t} \bar{D}=-\frac{\bar{F}_{i+1 / 2}^{D, H L L E}-\bar{F}_{i-1 / 2}^{D, H L L E}}{\Delta r},
$$

which is the expression (B.3) for the derivative of the flu associated to the variable $D$, centered at the inter-cell boundary located at $r_{i}$ with $\Delta r=\Delta x / 2$. Similarly happens for $J_{r}$ and $\tau$.

MoL for the geometry. The equations for the geometry do not use a FV discretization, instead use finit differences, which in practice translates into using expressions of derivatives for $r h s$ directly from (B.3 B.5), a discretization called vertex-centered, as opposed to cell-centered used for matter.

We also use the stencil (B.3) for the firs order spatial derivatives on the right hand sides of the equations for $\gamma_{r r}, \gamma_{\theta \theta}, K_{r r}, K_{\theta \theta}$ and the Chistoffel symbols, unless we suspect possible causal disconnection, which is a very important issue in Numerical Relativity.

Causal disconnection is related to advection terms in the right hand sides of the equations. These are terms of the type $\beta^{r} \partial_{r} g$ in (41 44), with $\beta^{r}$ the radial component of the shift, that we know, represents a local velocity of coordinates, from one spatial hypersurface to the next one. This define a causality restriction on the time integration with MoL.

For illustration consider again the case (B.6) with $a=\beta^{r}$ at a particular point of the domain, and $x=r$. The value of $\beta^{r}$ can be positive and big enough that the point $\left(t^{n}, r_{i-1}\right)$ may be causally disconnected from $\left(t^{n+1}, r_{i}\right)$ in the top plot of Fig. B.1. This problem is solved using the one-sided expressions (B.4) for the spatial derivative that uses only points to the right from $\left(t^{n}, r_{i}\right)$. The oposite case of $\beta^{r}<0$ can be solved using the one-sided formula (B.5) that uses only points to the left from $x_{i}$. The molecules illustrating these two possibilities are shown in the middle and bottom panel of Fig. B.1.

Summarizing, in practice the advection terms are handled explicitly for this example as follows

$$
\begin{aligned}
& \left.\beta^{r} \frac{\partial g}{\partial x}\right|_{\left(t^{n}, x_{i}\right)}=\left.\frac{-3 g_{i}^{n}+4 g_{i+1}^{n}-g_{i+2}^{n}}{2 \Delta x} \beta^{r}\right|_{\left(t^{n}, x_{i}\right)}, \beta^{r}>0, \\
& \left.\beta^{r} \frac{\partial g}{\partial x}\right|_{\left(t^{n}, x_{i}\right)}=\left.\frac{g_{i+1}^{n}-g_{i-1}^{n}}{2 \Delta x} \beta^{r}\right|_{\left(t^{n}, x_{i}\right)}, \beta^{r}=0, \\
& \left.\beta^{r} \frac{\partial g}{\partial x}\right|_{\left(t^{n}, x_{i}\right)}=\left.\frac{3 g_{i}^{n}-4 g_{i-1}^{n}+g_{i-2}^{n}}{2 \Delta x} \beta^{r}\right|_{\left(t^{n}, x_{i}\right)}, \quad \beta^{r}<0,
\end{aligned}
$$

We use the evolution equation for $\gamma_{r r}$ (41) to illustrate the semi-discrete version of one of the ADM equations that reads:

$$
\begin{aligned}
\left.\frac{\partial \gamma_{r r}}{\partial t}\right|_{\left(t^{n}, x_{i}\right)} & =-\left.2 \alpha K_{r r}\right|_{\left(t^{n}, x_{i}\right)} \\
& +\left.2 \gamma_{r r}\right|_{\left(t^{n}, x_{i}\right)} \frac{\beta_{i+1}^{r n}-\beta_{i-1}^{r n}}{2 \Delta r}+\text { advec },
\end{aligned}
$$

where

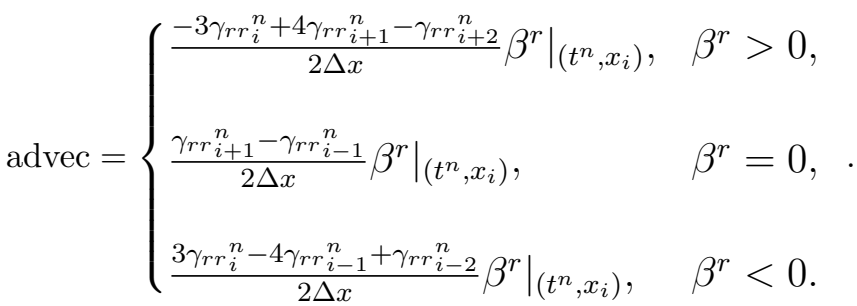

The firs term in the rhs is a function, the second term includes a centered derivative of $\beta^{r}$ and the third term is an advection term that uses one-sided molecules.

The implementation of derivatives in boundary conditions for geometry (57) also uses the one-sided stencil (B.5) for the right hand sides at $r_{\max }=N_{r}$.

Finally, there are also second order spatial derivatives in the right hand side of equations (41,44). Second order derivatives are not used at the boundaries, then we only need the centered expression, which for the generic function $g$ reads

$$
\left.\frac{\partial^{2} g}{\partial x^{2}}\right|_{\left(x_{i}, t^{n}\right)}=\frac{g_{i+1}^{n}-2 g_{i}^{n}+g_{i-1}^{n}}{\Delta x^{2}}+\mathcal{O}\left(\Delta x^{2}\right)
$$

which completes the details of the MoL, needed to reproduce the results in this paper. 


\section{Units}

In the calculations we use geometric units $G=c=1$. In these units, both space and time coordinates are in units of $M$, which is the scale that fi es the units of a particular physical system.

In order for the results to be eventually compared with observations, it is important to present the results using physical units. Following [6], conversion factors from geometric units to cgs units for length, time, mass, velocity, density and pressure are

$$
\begin{aligned}
r_{\mathrm{cgs}} & =1.47651 \times 10^{5}\left(\frac{M}{M_{\odot}}\right) r_{\mathrm{geo}} \\
t_{\mathrm{cgs}} & =4.92513 \times 10^{-6} c\left(\frac{M}{M_{\odot}}\right) t_{\mathrm{geo}} \\
m_{\mathrm{cgs}} & =1.9884 \times 10^{33}\left(\frac{G}{c^{2}}\right)\left(\frac{M}{M_{\odot}}\right) m_{\mathrm{geo}} \\
v_{\mathrm{cgs}} & =2.99792458 \times 10^{10}\left(\frac{1}{c}\right) v_{\mathrm{geo}} \\
\rho_{\mathrm{cgs}} & =6.17714 \times 10^{17}\left(\frac{G}{c^{2}}\right)\left(\frac{M_{\odot}}{M}\right)^{2} \rho_{\mathrm{geo}} \\
p_{\mathrm{cgs}} & =5.55173 \times 10^{38}\left(\frac{G}{c^{4}}\right)\left(\frac{M_{\odot}}{M}\right)^{2} p_{\mathrm{geo}},
\end{aligned}
$$

where the mass $M$ has to be given in solar masses. In the examples of the text we set $M=10^{6} M_{\odot}$ in the test fiel case and $M=100 M_{\odot}$ in the non-linear case.

\section{Geometrical quantities of the EF space-time}

The components of the extrinsic curvature for the EF metric are useful and here we construct their values. Starting from the fact that $\gamma_{i j}=\operatorname{diag}\left(\gamma_{r r}, \gamma_{\theta \theta}, \sin ^{2} \theta \gamma_{\theta \theta}\right)$ with $\gamma_{r r}=(1+[2 M / r])$ and $\gamma_{\theta \theta}=r^{2}$, and using (33) one has

$$
K_{i j}=-\frac{1}{2 \alpha}\left[\partial_{t} \gamma_{i j}-D_{i} \beta_{j}-D_{j} \beta_{i}\right],
$$

where the covariant derivative $D_{i}$ is consistent with the 3metric $\gamma_{i j}$ of $\Sigma_{t}$. That is, we have to use the symbols (46) in what follows. Then

$$
\begin{aligned}
K_{r r}- & =-\frac{1}{2 \alpha}\left[\partial_{t} \gamma_{r r}-2 D_{r} \beta_{r}\right]=\frac{1}{\alpha}\left(\partial_{r} \beta_{r}-\Gamma_{r r}^{r} \beta_{r}\right) \\
& =-\frac{2 M}{r^{2}} \frac{1+\frac{M}{r}}{\sqrt{1+\frac{2 M}{r}}}, \\
K_{\theta \theta} & =-\frac{1}{2 \alpha}\left[\partial_{t} \gamma_{\theta \theta}-2 D_{\theta} \beta_{\theta}\right]=\frac{1}{\alpha}\left(\partial_{\theta} \beta_{\theta}-\Gamma_{\theta \theta}^{r} \beta_{r}\right) \\
& =\frac{2 M}{\sqrt{1+\frac{2 M}{r}}},
\end{aligned}
$$

are the components of the extrinsic curvature of the EF metric, useful in Eqs. (50) and (52).

\section{E. Pseudocode}

The implementation of a code with the methods described for the test-fiel and non-linear regime has a very intuitive flu diagram. The order of the implementation of each step described in the paper can be summarized in the following pseudocode.

\section{E.1 Test fluid case}

1. Defin numerical parameters for the numerical domain $N_{r}, N_{t}$ from Sec. 2.1.

2. Assign memory to one dimensional arrays of size $N_{r}$ for the radial coordinate $r$, primitive variables $\rho_{0}, v^{r}, e$, and $p$, conservative variables $D, J_{r}, \tau$ and their past values for the implementation of the MoL, flu es and sources in the system (15), metric functions $\alpha, \beta^{r}, \gamma_{r r}, \gamma_{\theta \theta}$, non-zero stress-energy tensor components (14), non-zero Christoffel symbols (29) and the auxiliary functions $W, h$ and $\gamma$.

! Initial conditions

3. Defin the Eddington-Finkelstein metric in terms of $r$ using formulas (28) and set Christoffel symbols (29).

4. Defin the adiabatic index $\Gamma$ and set initial conditions for the primitive variables of the fluid namely $\rho_{0}, v^{r}, p$, and through the equation of state (9) determine $e=$ $p /\left(\rho_{0}(\Gamma-1)\right)$ and therefore $h=1+e+p / \rho_{0}$.

5. Defin the initial conservative variables $D, J_{r}, \tau$ through formulas ( $(\overline{8})$.

6. Evolution loop to be executed $N_{t}$ times.

\section{1 !Runge-Kutta Loop.}

Do for the three intermediate steps of the third order Runge-Kutta

- Calculate the right hand sides for the evolution equations (15) following the recipe in section II-A.

- Update the values of $D, J_{r}, \tau$

6.2 Diagnostics, where the accretion rate is calculated using formula (32)

\subsection{Save data}

! Ends Evolution loop.

7. Ends program. 


\section{E.2 Non-linear accretion case}

In this case the number of evolution equations is bigger and setting up initial conditions is more elaborate. The following is the flu of instructions.

1. Defin numerical parameters for the numerical domain $N_{r}, N_{t}$ from Sec. 4.2.2.

2. Assign memory to one dimensional arrays of size $N_{r}$ for the radial coordinate $r$, primitive variables $\rho_{0}, v^{r}, e$, $p$, conservative variables $D, J_{r}, \tau$ and their past values for the implementation of the MoL, flu es and sources in the system (15), metric gauge functions $\alpha$ and $\beta^{r}$, ADM variables $\gamma_{r r}, \gamma_{\theta \theta}, K_{r r}, K_{\theta \theta}$ and their past values for the use of the MoL, non-zero stress energy components (13), non-zero Christoffel symbols (A.2), auxiliary functions $W, h$ and $\gamma$, diagnostics variables $\mathcal{H}, \mathcal{M}^{r}$ and all their terms in expressions $(48,49)$, so as the expansion function (61) and Misner-Sharp mass $M_{M S}$ (66).

! Initial conditions

3. Defin the adiabatic index $\Gamma$ and set initial conditions for the primitive variables of the flui $\rho_{0}, v^{r}, p$ in (53). With these calculate $e=p /\left(\rho_{0}(\Gamma-1)\right)$ and $h=1+e+p / \rho_{0}$.

4. Defin initial values for the conservative variables $D, J_{r}, \tau$ with formulas (8).

5. Implement initial conditions for the metric and extrinsic curvature components consistent with the constraints following the recipe in Sec. 4.1
6. Evolution loop to be done $N_{t}$ times.

\section{1 !Runge-Kutta Loop}

Do for the three intermediate steps of the third order Runge-Kutta

- Calculate the right hand sides for geometry according to expressions (41.44).

- Calculate the right hand sides for the construction of null rays (65).

- Calculate the right hand sides for Euler equations (15).

- Update the values of hydrodynamical and geometric variables $D, J_{r}, \tau, \gamma_{r r}, \gamma_{\theta \theta}, K_{r r}, K_{\theta \theta}$

6.2 Diagnostics including location of the Apparent Horizon radius, Misner-Sharp mass $M_{M S}$, apparent horizon mass $M_{A H}$, violation of the constraints $\mathcal{H}, \mathcal{M}^{r}$, and their $L_{2}$ norm.

6.3 Save data

Ends Evolution loop.

7. Ends program.

We programmed our codes in Fortran 90.

\section{Acknowledgments}

This research is supported by grant CIC-UMSNH-4.9.
1. K. Akiyama et al. (The Event Horizon Telescope Collaboration), First M87 Event Horizon Telescope Results. I. The Shadow of the Supermassive Black Hole, Astrophys. J. Lett. 875 (2019) L1; Ibid., Astrophys. J. Lett. 875 (2019) L2; Ibid., Astrophys. J. Lett. 875 (2019) L3; Ibid., Astrophys. J. Lett. 875 (2019) L4; Ibid., Astrophys. J. Lett. 875 (2019) L5; Ibid., Astrophys. J. Lett. 875 (2019) L6.

2. J. A. González, F. S. Guzmán, Classificatio of a black hole spin out of its shadow using support vector machines, Phys. Rev. D 99 (2019) 103002, https: / /doi.org/10.1103/ PhysRevD.99.103002

3. C. W. Misner, K. S. Thorne, and J. A. Wheeler, Gravitation (W. H. Freeman and Company, San Francisco, 1973).

4. J. A. Font, J. M. Ibañez, A. Marquina, and J. M Martí, Multidimensional relativistic hydrodynamics: characteristic fields and modern high-resolution shock-capturing schemes, Astron. Astrophys. 282 (1994) 304. http: / / articles.adsabs. harvard.edu/pdf/1994A\%26A...282..304F

5. F. Banyouls, J. A. Font, J. M. Ibañez, L. Ma. Marí, J. A. Miralles, Numerical $3+1$ General Relativistic Hydrodynamics:
A Local Characteristic Approach, Approach, Astrophys, 476 (1997) 221.https://doi.org/10.1086/303604

6. L. Rezzolla and O. Zanotti, Relativistic Hydrodynamics (Oxford University Press, Oxford, 2013), https://doi.org/ 10.1093/acprof:oso/9780198528906.001.0001

7. F. S. Guzmán, F. D. Lora-Clavijo, and M. D. Morales, Revisiting spherically symmetric relativistic hydrodynamics, Rev. Mex. Fís. E 58 (2012) 84. https://rmf.smf.mx/ojs/ rmf-e/article/view/4683

8. J. M. Martí and E. Müller, Numerical Hydrodynamics in Special Relativity, Living Rev. Relativ. 6 (2003) 7, https:// doi.org/10.12942/lrr-2003-7

9. J. W. Thomas. Numerical Partial Differential Equations: Conservation Laws and Elliptic Equations (Springer-Verlag, New York, 1999), https://doi.org/10.1007/ 978-1-4612-0569-2

10. R. L. LeVeque, Numerical Methods for Conservation Laws (Birkhäuser, Basel, 1992), https://doi.org/10.1007/ 978-3-0348-8629-1

11. E. F. Toro, Riemann Solvers and Numerical Methods for Fluid 
Dynamics (Springer-Verlag, Berlin, 1999), https://doi. org/10.1007/b79761

12. P. Harten, B. Lax, B. B. van Leer, On Upstream Differencing and Godunov-Type Schemes for Hyperbolic Conservation Laws, Soc. Ind. Appl. Math. Rev. 25 (1983) 294, https: //doi.org/10.1137/1025002.

13. B. Einfeldt, On godunov-type methods for gas dynamics, $J$. Comp. Phys. 25 (1988) 294. https: / / epubs . siam.org/ doi/10.1137/0725021

14. A. Cruz-Osorio, A. González-Juárez, F. S. Guzmán, F. D. LoraClavijo. Numerical solution of the wave equation on particular space-times using CMC slices and scri-fixing conformal compactification, Rev. Mex. Fis. 56 (2010) 456. https: //rmf. smf.mx/pdf/rmf/56/6/56_6_456.pdf

15. J. Thornburg, A $3+1$ Computational Scheme for Dynamic Spherically Symmetric Black Hole Spacetimes - II: Time Evolution. arXiv:gr-qc/9906022.

16. F. S. Guzmán, L. Lehner, O. Sarbach, Do unbounded bubbles ultimately become fenced inside a black hole?, Phys. Rev. D 76 (2007) 066003. https: //doi.org/10.1103/ PhysRevD.76.066003

17. H. Bondi, On spherically symmetrical accretion, Mon. Not. R. Astron. Soc. 112 (1952) 195, https://doi.org/10. $1093 / \mathrm{mnras} / 112.2 .195$

18. F. C. Michel. Accretion of matter by condensed objects, Astro. Space Sci. 15 (1972) 153. https://doi.org/10.1007/ BF00649949.

19. P. Papadopoulos and J. A. Font, Relativistic Hydrodynamics around Black Holes and Horizon Adapted Coordinate Systems, Phys. Rev. D 58 (1998) 024005 https://doi.org/10. $1103 /$ PhysRevD.58.024005

20. M. Gracia-Linares and F. S. Guzmán, Accretion of supersonic winds onto black holes in 3D: stability of the shock cone, ApJ 812 (2015) 23, https://doi.org/10.1088/ $0004-637 \mathrm{X} / 812 / 1 / 23$.

21. F. D. Lora-Clavijo. Dr. Sc. Thesis. Universidad Michoacana de San Nicolás de Hidalgo, 2013. http://www. ifm.umich. $\mathrm{mx} / \sim$ guzman/LoraClavijoDrScThesis.pdf

22. M. Alcubierre, Introduction to $3+1$ Numerical Relativity, (Oxford Science Publications 2008). https://doi.org/10. 1093/acprof:oso/9780199205677.001.0001
23. T. W. Baumgarte and S. L. Shapiro, Numerical Relativity (Cambridge University Press, Cambdirge, 2010), https://doi. org/10.1017/CB09781139193344.

24. I. Avilés, G. Estrada, J. A. González, F. S. Guzmán Pseudospectral vs Finite Differences methods in Numerical Relativity, Rev. Mex. Fis. E. 63 (2017) 25, https : //rmf . smf . $\mathrm{mx} / \mathrm{ojs} / \mathrm{rmf}-\mathrm{e} / \mathrm{article/view/4765/4671}$

25. M. Campanelli, C. O. Lousto, P. Marronetti, Y. Zlochower, Accurate Evolutions of Orbiting Black-Hole Binaries without Excision, Phys. Rev. Lett. 96 (2006) 111101 https : / doi . org/10.1103/PhysRevLett.96.111101

26. J. G. Baker, J. Centrella, D. Choi, M. Koppitz, J. van Meter, Gravitational-Wave Extraction from an Inspiraling Configuration of Merging Black Holes, Phys. Rev. Lett. 96 (2006) 111102. https://doi.org/10.1103/PhysRevLett. 96.111102

27. F. S. Guzmán, In preparation.

28. J. W. Thomas, Numerical Partial Differential Equations: Finite Difference Methods (Springer-Verlag, New York, 1995), https://doi.org/10.1007/978-1-4899-7278-1

29. R. A. Matzner et al., Geometry of a Black Hole Collision, Science 270 (1995) 941. https: / / science. sciencemag. org/content/270/5238/941

30. https://science.sciencemag.org/content/ $270 / 5238 / 941$

31. http://www. cactuscode.org

32. P. Diener, A new general purpose event horizon finde for 3D numerical spacetimes, Class. Quantum Grav. 20 (2003) 4901, https://doi.org/10.1088/0264-9381/20/ $22 / 014$.

33. F. S. Guzmán, L. Lehner, O. Sarbach, Do unbounded bubbles ultimately become fenced inside a black hole? Phys. Rev. D 76 (2007) 066003. https: //doi.org/10.1103/ PhysRevD.76.066003

34. C. W. Misner, D. H. Sharp, Relativistic Equations for Adiabatic, Spherically Symmetric Gravitational Collapse, Phys. Rev. 136 (1964) B571, https://doi.org/10.1103/PhysRev. 136. B571 